\author{
Sajjad Seyedsalehi \\ Department of Mechanical Engineering, \\ Michigan State University, \\ East Lansing, MI 48824-1226 \\ Liangliang Zhang \\ Department of Statistics and Probability, \\ Michigan State University, \\ East Lansing, Ml 48824-1027 \\ Jongeun Choi \\ Department of Mechanical Engineering. \\ Department of Electrical and \\ Computer Engineering, \\ Michigan State University, \\ East Lansing. Ml 48824-1226

Seungik Baek
Department of Mechanical Engineering,
Michigan State University,
2457 Engineering Building,
East Lansing, Ml 48824-1226
e-mail: sbaek@egr.msu.edu

\title{
Prior Distributions of Material
}

Parameters for Bayesian Calibration of Growth and Remodeling Computational Model of Abdominal Aortic Wall

For the accurate prediction of the vascular disease progression, there is a crucial need for developing a systematic tool aimed toward patient-specific modeling. Considering the interpatient variations, a prior distribution of model parameters has a strong influence on computational results for arterial mechanics. One crucial step toward patient-specific computational modeling is to identify parameters of prior distributions that reflect existing knowledge. In this paper, we present a new systematic method to estimate the prior distribution for the parameters of a constrained mixture model using previous biaxial tests of healthy abdominal aortas (AAs). We investigate the correlation between the estimated parameters for each constituent and the patient's age and gender; however, the results indicate that the parameters are correlated with age only. The parameters are classified into two groups: Group-I in which the parameters $c_{e}, c_{k_{1}}, c_{k_{2}}, c_{m_{2}}$, $G_{h}^{c}$, and $\phi^{e}$ are correlated with age, and Group-II in which the parameters $c_{m_{l}}, G_{h}^{m}, G_{l}^{e}, G_{2}^{e}$, and $\alpha$ are not correlated with age. For the parameters in Group-I, we used regression associated with age via linear or inverse relations, in which their prior distributions provide conditional distributions with confidence intervals. For Group-II, the parameter estimated values were subjected to multiple transformations and chosen if the transformed data had a better fit to the normal distribution than the original. This information improves the prior distribution of a subject-specific model by specifying parameters that are correlated with age and their transformed distributions. Therefore, this study is a necessary first step in our group's approach toward a Bayesian calibration of an aortic model. The results from this study will be used as the prior information necessary for the initialization of Bayesian calibration of a computational model for future applications. [DOI: 10.1115/1.4031116]

Keywords: abdominal aortic aneurysm, model parameters, $G \& R$ model, correlation, age

\section{Introduction}

Computational models of vascular growth and remodeling $(G \& R)$ have emerged in predictive medicine with an increasing range of applications in arterial adaptation from physiological conditions, aging, and vascular diseases. As our understanding of the mechanobiological physiology and vascular diseases accumulates [1,2], the G\&R computational models are able to more accurately account for mechanisms involved in vascular disease progression. While the computational models have advanced in capturing the main bio-chemo-mechanical processes [3-5], it is now a critical question as to how we can determine multiple parameters for a given patient [6], in that it does not take into account various uncertainties during the progression of the disease and variability among patients in clinics.

Rupture of abdominal aortic aneurysms (AAAs) results in high mortality rates of $80-90 \%$ [7-10]. Computational models of AAAs are indispensable research tools for describing vascular material behaviors for providing a prediction capability. Finite element models (FEMs) take into account not only the subjectspecific geometry but also the material properties of the aorta $[11,12]$, and utilize stress and strength as the key parameters for

\footnotetext{
${ }^{1}$ Corresponding author.

Manuscript received January 7, 2015; final manuscript received July 6, 2015; published online August 6, 2015. Assoc. Editor: Jonathan Vande Geest.
}

estimating the rupture risk and severity of the aneurysm [13,14]. Recently, several groups have been actively researching the accurate estimation of AAA's wall stress using computational models such as FEM classical models [15-18] and FEM-G\&R models [19-24].

Several strain energy models for the aorta have been introduced so far. Some of these models use a phenomenological macroscopic approach and consider the aorta as a uniform material $[25,26]$. Particularly, structurally defined strain energy functions introduced by Holzapfel et al. [27,28] are popular in arterial mechanics because they describe the mechanical behavior well for various arteries and can account for fibers distributed in multiple directions in the arterial wall. These strain energy functions and their variations include two parts: an isotropic behavior represented by elastin and anisotropic behavior presented by multiple collagen fiber families used for basilar, carotid arteries, and human aorta [29-31]. In the multiple-fiber models, increasing the number of parameters provides more flexibility and generally produces better fitting, but more than six fiber families can cause a bias error and an overfitting problem [32]. For human healthy and aneurysmal AAs, Ferruzzi et al. [33] found that a four-fiber family model can capture the biaxial mechanical behavior. However, those studies did not consider the prestretch and multiple constituents that can be modeled independently. Instead, constrained mixture models have been developed, in which the arterial wall is considered to be a mixture of three constituents, namely elastin, 
collagen fiber families, and smooth muscle cells, independently with different stress-free configurations. For more details about the constrained mixture models, refer to Refs. [19,34,35].

The G\&R computational models of the aorta have been further implemented in the patient-specific modeling [11,36-38], particularly in AAA modeling. Initially, the studies focused on the growth mechanism by which the aortic wall alters collagen turnover, homeostasis, and remodeling. It is, however, a critical step for determining the subject-specific model parameters considering the wide range of material parameters such as material stiffness, prestretch, anatomical geometry, wall composition, and ultimately providing an accurate prediction for a specific clinical application. Wilson et al. [6] used a constrained mixture model with four fiber families and reinforced the importance of model parameters on the G\&R results. They estimated parameters including prestretches using a nonlinear regression with a weighted penalty method for four nonaneurysmal aortas; ages ranging from 47 to 69 . He used data from an experimental biaxial tension test performed by Vande Geest et al. [39]. The computational model initiated AAAs by inducing elastin degradation. Their results showed that the parameters of the nonaneurysmal aortas from different subjects have a strong influence on the mechanical behavior, the aneurysm expansion rate and the geometric parameters. Although both Wilson et al. [6] and Roccabianca et al. [40] captured the mechanical behaviors and have similar values for parameters, there are inconsistencies among multiple groups, even though the same data from Vande Geest et al. [39] were used. Hence, the motivation is to examine how the inconsistencies were arisen from and what can be done to provide a better prior distribution (distribution for model parameters) to reflect the mechanical behavior. We propose a systematic method to determine relevant prior distributions and take into account both population-based parameters and subject-specific parameters.

In this study, a systematic method is developed to construct prior conditional distributions of material parameters of the AA at the given age and gender. First, the G\&R model is briefly described and the constrained mixture approach for aortic mechanical behavior is introduced. Second, the parameters of multiple constituents are estimated by using the biaxial test data provided in the literature [41]. Next, using a regression study, for each parameter we decide if it is correlated to age and/or gender. For parameters with correlations to age and/or gender, conditional normal distributions are constructed for given functions of age and gender. The rest of the parameters which are not correlated to either age or gender are modeled by normal distribution fitted to either the original values or transformed values, depending on the test's $P$-values. Results of the prior distributions for three ages of 45, 60, and 75 years are illustrated and compared with other simple approaches.

Finally, we capitalize on the importance of the prior distributions in the Bayesian approach as the first and necessary step for our future goal of patient specific modeling for clinical applications.

\section{Methods}

2.1 Constitutive Model. Previously, a constraint mixture model was introduced and utilized by many groups, including our own, for modeling the nonlinear mechanical behavior of the human aortic wall [36]. In this model, we assume that the aortic wall consists of three different constituents, $i$, including elastin $(i=e)$; four collagen fiber families $(i=1, \ldots, k, \ldots, 4)$; and smooth muscle cells $(i=m)$. Elastin is an isotropic material that dominates the aortic wall, while collagen fibers and smooth muscle cells are aligned in the aortic wall. Specifically, smooth muscle cells are aligned circumferentially and four collagen fiber families are aligned in the axial $(i=1)$, circumferential $(i=2)$, and two diagonal $(i=3,4)$ directions.

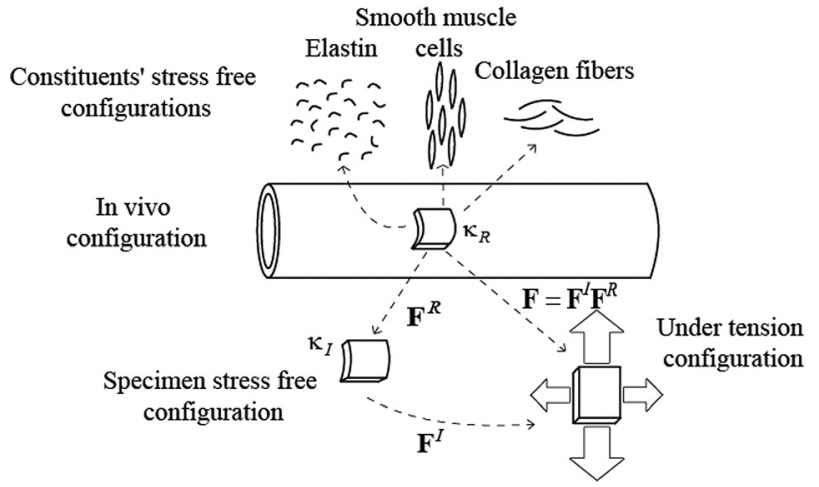

Fig. 1 Different configurations of the cut specimen and constituents of the aorta

We assume the prestressed in vivo aorta as the reference configuration, $\kappa_{R}$, while a traction-free, cut specimen is considered as the intermediate configuration, $\kappa_{I}$ (see Fig. 1). $\mathbf{F}^{R}$ and $\mathbf{F}^{I}$ are the $2 \mathrm{D}$ deformation gradient tensors mapping from $\kappa_{R}$ to $\kappa_{I}$ and from $\kappa_{I}$ to $\kappa$ (under biaxial tension), respectively. We have $\mathbf{F}^{R}=$ $\operatorname{diag}\left\{F_{\theta}^{R}, F_{z}^{R}\right\}$ and $\mathbf{F}^{I}=\operatorname{diag}\left\{F_{\theta}^{I}, F_{z}^{I}\right\} . F_{\theta}^{I}$ and $F_{z}^{I}$ are the stretches in the circumferential and axial directions, respectively, which have been measured during experiments. Additionally, the 2D deformation gradient from the prestressed in vivo (reference) configuration, $\kappa_{R}$, to the current (under tension) configuration, $\kappa$, is given by $\mathbf{F}=\mathbf{F}^{I} \mathbf{F}^{R}=\operatorname{diag}\left\{\lambda_{\theta}, \lambda_{z}\right\}$ and the right Cauchy-Green tensor is $\mathbf{C}=\mathbf{F}^{\mathrm{T}} \mathbf{F}$.

We assume a different stress-free configuration for each constituent named, natural configuration, $\kappa_{N}$. The stretches of collagen fiber families and smooth muscle cells from their own natural configurations to the current configuration are written as

$$
\begin{aligned}
& \lambda_{n}^{k}=G_{h}^{c} \lambda^{k} \\
& \lambda_{n}^{m}=G_{h}^{m} \lambda_{\theta}
\end{aligned}
$$

where $G_{h}^{c}$ and $G_{h}^{m}$ are the homeostatic stretches of collagen and smooth muscle cells, respectively. We have assumed the same stretch for all four fiber families [7,11]. $\lambda^{k}$ is the stretch of $k$ th fiber family from $\kappa_{R}$ to $\kappa$ and is given as

$$
\lambda^{k}=\sqrt{\left(\lambda_{\theta} \sin \alpha^{k}\right)^{2}+\left(\lambda_{z} \cos \alpha^{k}\right)^{2}}
$$

where $\alpha^{k}$ is the angle between the $k$ th collagen fiber family and the one in the axial direction. $\alpha^{k}$ takes the values of $\alpha^{1}=90 \mathrm{deg}, \alpha^{2}=0 \mathrm{deg}$, and $\alpha^{3}=\alpha^{4}=\alpha$, for the collagen fiber families aligning along the axial, circumferential, and two diagonal directions, respectively. In previous studies [6,11], $\alpha$ was assumed as $45 \mathrm{deg}$ fixed, but in other studies [33,40] $\alpha$ was estimated in their parameter estimations. By treating $\alpha$ as an unknown in the parameter estimation, the anisotropic mechanical behavior is taken into account for the collagen fiber families.

Additionally, the 2D homeostatic stretch tensor of elastin $\mathbf{G}^{e}$ which is the mapping from $\kappa_{N}$ to $\kappa_{R}$, is written as

$$
\mathbf{G}^{e}=\operatorname{diag}\left\{G_{1}^{e}, G_{2}^{e}\right\}
$$

where $G_{1}^{e}$ and $G_{2}^{e}$ are elastin's homeostatic stretches in circumferential and axial direction, respectively. The tensors of elastin, from its natural configuration $\kappa_{N}$ to the current configuration (under tension), are defined as

$$
\begin{gathered}
\mathbf{F}_{n}^{e}=\mathbf{F G}^{e} \\
\mathbf{C}_{n}^{e}=\mathbf{F}_{n}^{e^{\mathrm{T}}} \mathbf{F}_{n}^{e}=\mathbf{G}^{e^{\mathrm{T}}} \mathbf{C G}^{e}
\end{gathered}
$$


For constituents, the stored energy functions per unit mass are given by

$$
\begin{gathered}
\psi^{e}\left(\mathbf{C}_{n}^{e}\right)=\frac{c_{e}}{2}\left\{C_{n[11]}^{e}+C_{n[22]}^{e}+\frac{1}{C_{n[11]}^{e} C_{n[22]}^{e}-C_{n[12]}^{e}}-3\right\} \\
\psi^{k}\left(\lambda_{n}^{k}\right)=\frac{c_{k_{1}}}{4 c_{k_{2}}}\left\{\exp \left[c_{k_{2}}\left(\lambda_{n}^{k^{2}}-1\right)^{2}\right]-1\right\} \\
\psi^{m}\left(\lambda_{n}^{m}\right)=\frac{c_{m_{1}}}{4 c_{m_{2}}}\left\{\exp \left[c_{m_{2}}\left(\lambda_{n}^{m^{2}}-1\right)^{2}\right]-1\right\}
\end{gathered}
$$

for elastin, collagen fiber families, and smooth muscle, respectively. $c_{e}, c_{k_{1}}, c_{k_{2}}, c_{m_{1}}$, and $c_{m_{2}}$ are intrinsic material parameters, and $C_{n[i j]}^{e}$ are components of $\mathbf{C}_{n}^{e^{2}}$.

In addition to the above passive energy functions, a smooth muscle cell is associated with an active muscle tone for the energy per unit mass given as [41]

$$
\psi_{\mathrm{act}}^{m}=\frac{S}{\rho_{R}}\left\{\lambda_{\theta}+\frac{1}{3} \frac{\left(\lambda_{M}-\lambda_{\theta}\right)^{3}}{\left(\lambda_{M}-\lambda_{0}\right)^{2}}\right\}
$$

where $\lambda_{M}$ and $\lambda_{0}$ are the stretches at which the active force is at the maximum and at zero, respectively. $S$ is the stress when the smooth muscle has its peak contraction and $\rho_{R}$ is the density of the aortic wall. We set the values as $\lambda_{M}=1.4, \lambda_{0}=0.8$, $S=54 \mathrm{kPa}$, and $\rho_{R}=1050 \mathrm{~kg} \mathrm{~m}^{-3}[6]$.

The passive and active stored energy functions for the aortic wall per unit of mass are given by

$$
\begin{gathered}
\Psi_{\mathrm{pas}}=\phi^{e} \psi^{e}+\phi^{m}\left(\psi^{m}\right)+\sum_{k=1}^{4} \phi_{k}^{c} \psi^{k} \\
\Psi_{\text {act }}=\phi^{m} \psi_{\text {act }}^{m}
\end{gathered}
$$

where $\phi^{e}$ and $\phi^{m}$ are the elastin and smooth muscle mass fractions, respectively. $\phi_{k}^{c}\left(k\right.$ th element of matrix $\left.\phi^{\mathbf{c}}(k)\right)$ is the mass fraction of $k$ th collagen fiber families where $\phi^{\mathbf{c}}=[0.1,0.1$, $0.4,0.4]\left(1-\phi^{e}-\phi^{m}\right) . \quad \phi^{e}$ is estimated in our estimation approach, while similar to the previous studies $[6,36,42]$ for the contribution of smooth muscle cells a pre-assigned value of 0.15 is used for $\phi^{m}$. For the membrane model, the passive and active membrane stress (tension) can be calculated by [36]

$$
\begin{gathered}
\mathbf{T}_{\mathrm{pas}}=\frac{2}{J} \mathbf{F} \frac{\partial w_{\mathrm{pas}}}{\partial \mathbf{C}} \mathbf{F}^{\mathrm{T}} \\
T_{\mathrm{act}}=\frac{1}{\lambda_{z}} \frac{\partial w_{\mathrm{act}}}{\partial \lambda_{\theta}}
\end{gathered}
$$

where $w_{\text {pas }}=M_{R} \Psi_{\text {pas }}$ for the passive strain energy function and $w_{\text {act }}=M_{R} \Psi_{\text {act }}$ for the active strain energy function per unit reference area, with $M_{R}$ being the total wall mass density per unit reference area and $J=\operatorname{det} \mathbf{F}$. Finally, the overall membrane stress in the wall is

$$
\mathbf{T}=\mathbf{T}_{\mathrm{pas}}+T_{\mathrm{act}} \mathbf{e}_{\theta} \otimes \mathbf{e}_{\theta}
$$

Considering that $\kappa_{I}$ is the traction free configuration, $\mathbf{T}=0$ for $\mathbf{F}=\mathbf{F}^{R}$. In order to calculate the Cauchy stress, the membrane stress must be divided by the wall thickness $h$ (under tension) which by the incompressibility assumption can be calculated as

$$
h=\frac{M_{R}}{\rho_{R} J}
$$

We have 11 unknown parameters, which we will estimate using nonlinear regression. These parameters are the intrinsic material parameters $c_{e}, c_{k_{1}}, c_{k_{2}}, c_{m_{1}}, c_{m_{2}}$, the prestretches of constituents $G_{h}^{c}, G_{h}^{m}, G_{1}^{e}, G_{2}^{e}$, the elastin mass fraction $\phi^{e}$, and the angle of the diagonal collagen fiber family $\alpha$ with respect to the axial direction.

2.2 Nonlinear Regression. We estimate model parameters for 17 subjects using experimental data provided by Vande Geest et al. [39,43]. They have studied specimens harvested from 21 healthy human AAs for a wide range of ages of 19-78 years old. We did not incorporate the data of four specimens in our study. The reason for this is that age and gender information were not provided for two of the subjects and test data was highly noisy for the other two specimens. The biaxial tests performed in the experiment account for seven sets of axial to circumferential tension ratios: 1:0.1, 1:0.5, 1:0.75, 1:1, 0.75:1, 0.5:1, and 0.1:1. We utilize the stress-stretch results for parameter estimation. The best-fit parameters are obtained by minimizing the difference between the experimentally measured and theoretically calculated Cauchy stress for each specimen with the addition of penalty terms for the constituents' homeostatic stress values and aortic wall thickness.

Due to the nonlinear nature of the problem and the large number of unknown parameters, we need to carefully select the total number of estimation parameters; otherwise, the parameter estimation has a high cost in computation and results in multiple answers for each specimen (cf, overfitting problem [32]). Furthermore, in order to make the parameter estimation efficient, it is divided into two steps: (1) while five parameters are temporary fixed, the other parameters are estimated with penalty terms using the biaxial tests from Vande Geest et al. [39] and (2) the results of the first parameter estimation (for six parameters) are used as the initial values for the full parameter estimation. For the first step, we prescribe the fixed values for prestretches and the angle, $G_{1}^{e}, G_{2}^{e}, G_{h}^{c}, G_{h}^{m}$, and $\alpha$, which were obtained from the previous literature. The values are listed from the literature $[6,33,36,40,44]$ along with the averaged values in Table 1 . In the second step, all the fixed values and penalty functions are removed for the full parameter estimation, except the penalty terms for homeostatic stresses and aortic wall thickness. In vascular G\&R computational models, the stress-mediated mechanical homeostasis is generally assumed [34] but the exact values are not known. The thicknesses of aortic specimens are not available; therefore, we use the reported mean thickness of $1.4 \mathrm{~mm}$ [6]. We consider the thickness of the aorta and the average homeostatic stress in each constituent (elastin and collagen fibers) in the cost function as penalty terms, wherein the cost function minimizes the best-fit parameters with some tolerance. We set the homeostatic circumferential stress in elastin and collagen fiber families at $100 \mathrm{kPa}$ [6], and the homeostatic thickness of the aortic wall at $1.4 \mathrm{~mm}$ [6]. The goal function $e$ is defined as

Table 1 Prestretches and the angle between axial and diagonal fibers for five studies in the literature. These values are averaged and fixed at the first step in the parameter estimation.

\begin{tabular}{lccccc}
\hline \hline Source & $G_{1}^{e}$ & $G_{2}^{e}$ & $G_{h}^{c}$ & $G_{h}^{m}$ & $\alpha(\mathrm{deg})$ \\
\hline Zeinali-Davarani et al. [44] & 1.22 & 1.23 & 1.034 & 1.165 & 45 \\
Zeinali-Davarani et al. [36] & 1.25 & 1.25 & 1.07 & 1.2 & 45 \\
Wilson et al. [6] & 1.29 & 1.30 & 1.05 & 1.10 & 45 \\
& 1.18 & 1.29 & 1.03 & 1.10 & 45 \\
& 1.34 & 1.25 & 1.06 & 1.10 & 45 \\
Roccabianca et al. [40] & 1.19 & 1.21 & 1.03 & 1.11 & 45 \\
Ferruzzi et al. [33] & 1.2 & 1.2 & 1.08 & - & 45.83 \\
Initial values & - & - & - & - & $\begin{array}{c}45.82 \\
\text { (averaged) } \\
\text { (1.24) }\end{array}$ \\
\hline \hline
\end{tabular}


Prior distribution of parameters
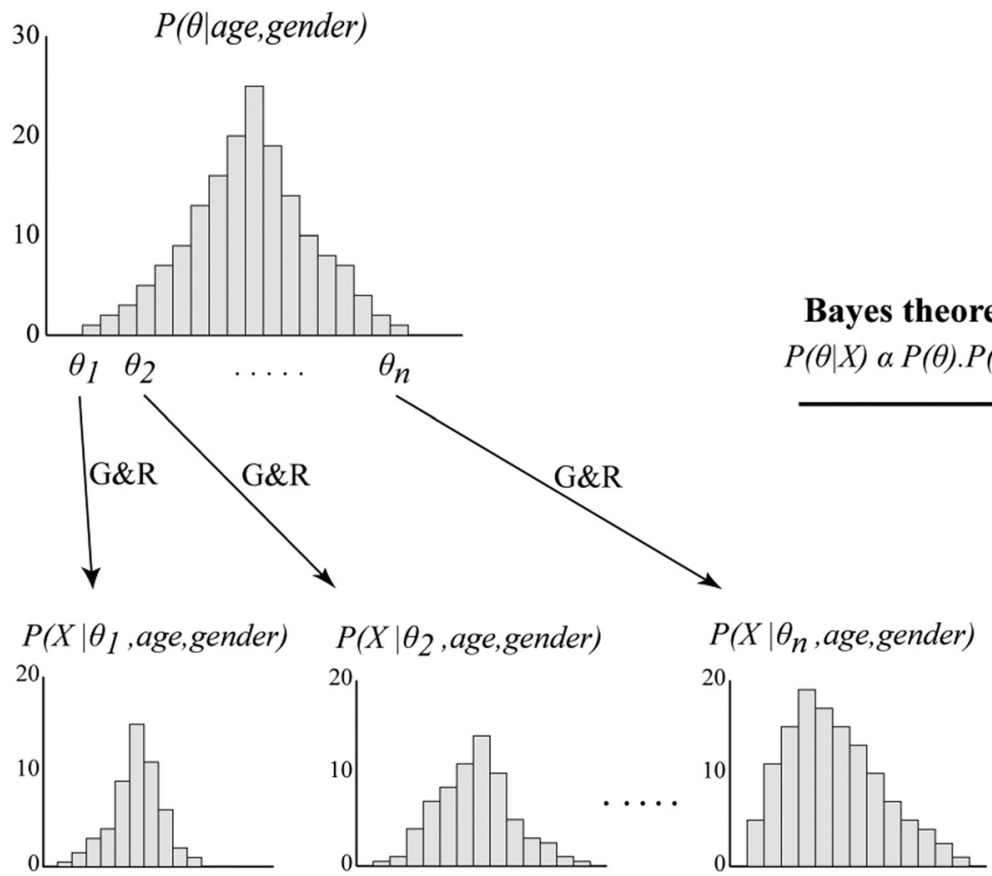

Posterior distribution of parameters

Bayes theorem
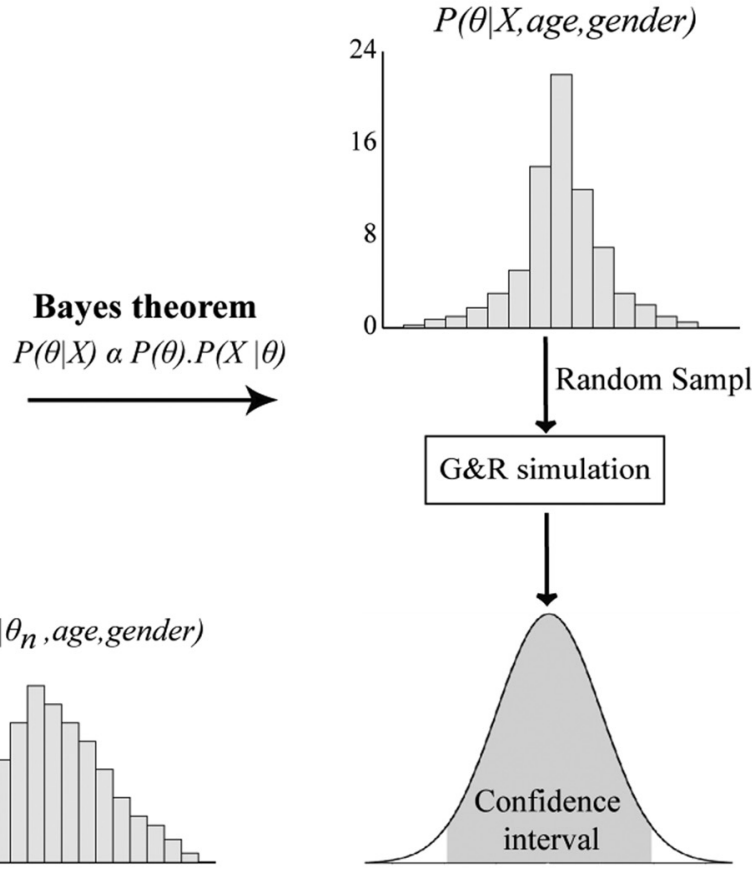

Fig. 2 Schematic drawing of Bayesian calibration of a G\&R model. $\boldsymbol{P}(\boldsymbol{\theta} \mid$ age, gender $), \boldsymbol{P}(\mathbf{X} \mid \boldsymbol{\theta}$, age, gender $)$, and $\boldsymbol{P}(\theta \mid \mathbf{X}$, age, gender) denote the conditional joint (prior) distribution, likelihood function, and posterior distribution of the model parameters, respectively.

$$
\begin{aligned}
e= & \sum_{\text {experiment }}\left\{\left(T_{z z}^{\mathrm{th}}-T_{z z}^{\mathrm{exp}}\right)^{2}+\left(T_{\theta \theta}^{\mathrm{th}}-T_{\theta \theta}^{\mathrm{exp}}\right)^{2}\right\}+a_{1}\left(t_{\theta \theta}^{h, e}-100\right)^{2} \\
& +a_{2}\left(t_{\theta \theta}^{h, c}-100\right)^{2}+a_{3}\left(\triangle^{\mathrm{th}}-0.0014\right)^{2}
\end{aligned}
$$

where

$$
\begin{gathered}
t_{\theta \theta}^{h, e}=\rho_{R} c_{e} G_{1}^{e 2}\left(1-\frac{1}{G_{1}^{e 4} G_{2}^{e 2}}\right) \\
t_{\theta \theta}^{h, c}=\frac{\rho_{R} c_{k_{1}}}{1-\phi^{m}-\phi^{e}}\left(G_{h}^{c 4}-G_{h}^{c 2}\right) \exp \left\{c_{k_{2}}\left(G_{h}^{c 2}-1\right)^{2}\right\} \\
\times \sum_{k=1}^{4} \phi_{k}^{c} \sin \left(\alpha^{k}\right)^{2}
\end{gathered}
$$

where $T_{z z}^{\text {th }}$ and $T_{z z}^{\exp }$ are theoretical and experimental axial stresses, respectively, and $T_{\theta \theta}^{\mathrm{th}}$ and $T_{\theta \theta}^{\exp }$ are theoretical and experimental circumferential stresses, respectively. $t_{\theta \theta}^{h, e}$ and $t_{\theta \theta}^{h, c}$ are theoretical homeostatic stresses (at the mean pressure) in circumferential direction in elastin and collagen fiber families, respectively. $\triangle^{\text {th }}$ is the calculated homeostatic thickness of the aortic wall. Adding these penalty terms reduces the uncertainty of our nonlinear parameter estimation and guides us toward a more reliable estimation. Coefficients $a_{1}$ and $a_{2}$ are chosen by trial and error so that the circumferential homeostatic stresses in elastin $\left(t_{\theta \theta}^{h, e}\right)$ and collagen $\left(t_{\theta \theta}^{h, c}\right)$ do not deviate from their expected values $(100 \mathrm{kPa})$ by more than $10 \%$. Additionally, coefficient $a_{3}$ is set to keep the estimated thickness $\left(\triangle^{\text {th }}\right)$ within $5 \%$ error from its reported value $(0.0014 \mathrm{~m})$. By trial and error, we set $a_{1}=a_{2}=10^{-3}$ and $a_{3}=2 \times 10^{7}$.

For the nonlinear regression minimization tool, we use the fminsearch function in MATLAB (Mathwork, Natick, MA). This function uses the Nelder-Mead simplex method for optimization. The goal function is to minimize $e$. We consider two criteria for stopping the optimization. Iteration is terminated if either the changes in $e$ or the changes in parameters are smaller than TolFun $=10^{-5}$ or TolX $=10^{-5}$, respectively.

2.3 Bayesian Approach for the G\&R Computational Model. This study is one step toward the ultimate goal of subjectspecific calibration of G\&R modeling. In this section, Bayesian calibration is briefly explained and the importance of prior distribution is interpreted. Calibration is the term used to describe the inverse process of fitting a model to data, referred to as inference $[45,46]$. We consider a problem in which we have a computer model (e.g., a G\&R computational model) of a physical system (aorta) along with observations of the system (real patient's CT scan images and experimental tests). The Bayesian model is a natural choice for the incorporation of prior information (assumed distribution of parameters), physical observations, and other information related to the model [45]. Using the Bayesian calibration, the posterior distributions are constructed and the calibrated parameters are evaluated based on those distributions. Then, we insert the calibrated parameter values of the computer model again to predict the growth of the AAA in a future time by using the predictive distribution in the Bayesian model. The framework of Bayesian calibration is briefly illustrated in Fig. 2.

The different stages of application of the Bayesian theorem are illustrated in Fig. 2. $P(\boldsymbol{\theta} \mid$ age, gender $)$ is the conditional joint distribution of the model parameters, which we aim to construct in the current study. The histograms illustrate the Bayesian approach more clearly; in reality, however, it is not possible to represent a joint distribution with more than two random variables other than showing the distribution for each variable separately. The prior distribution will be constructed with estimated parameters for the 17 healthy aortas mentioned previously. From the prior distribution, we perform multiple sampling $\left(\vec{\theta}_{1}, \vec{\theta}_{2}, \ldots, \vec{\theta}_{n}\right)$ and for each sample we run the G\&R simulation (e.g., pressure/flow induced vascular adaptation, aging, local AAA expansion) separately. One output of the G\&R is a distribution for the parameter $\vec{X}$. For 


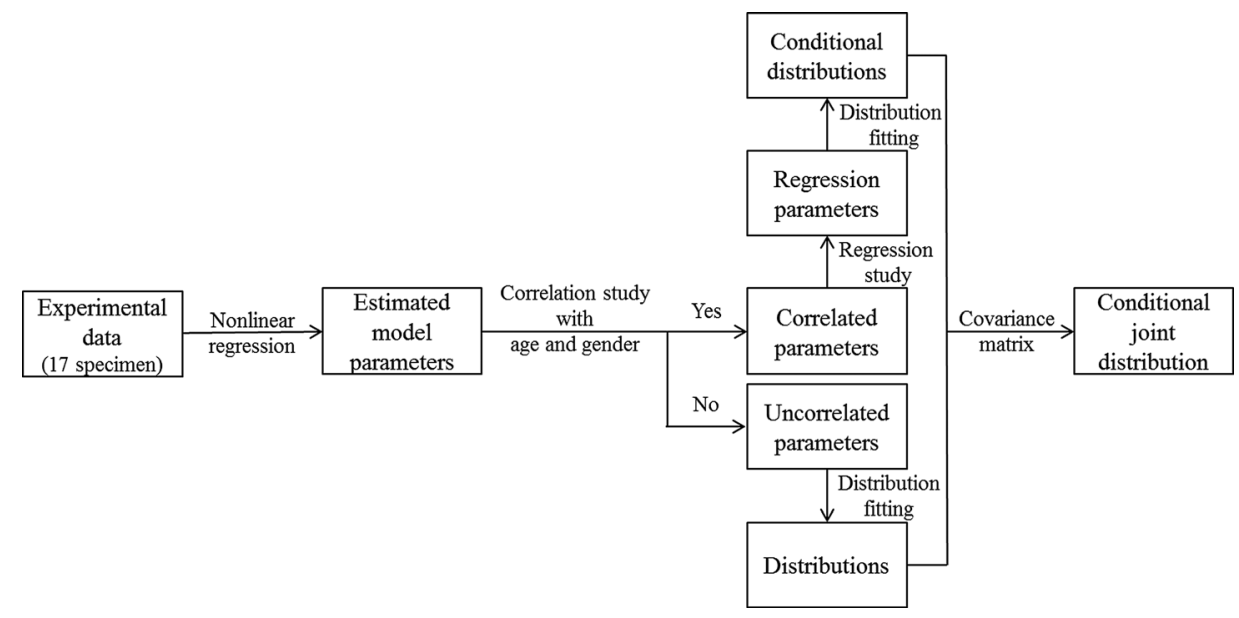

Fig. 3 Flow chart illustrating the steps involved in the construction of the prior distribution

example, $\vec{X}$ could be the maximum diameter of the AA that is measurable from the CT-scan image of the patient. Although we have chosen just one set of parameters for each simulation, due to the uncertainty modeled in the G\&R model, the output will have a distribution. Using these distributions, we construct the likelihood function $P(\mathbf{X} \mid \boldsymbol{\theta}$, age, gender $)$. The last stage is to use the Bayes theorem and build the posterior distribution for model parameters. The posterior distribution $P(\boldsymbol{\theta} \mid \mathbf{X}$, age, gender $)$ is more reliable than the prior distribution as it considers more patient-specific information (X, age, gender) while reducing the uncertainty of the prior distribution caused by the nonlinearity of the estimation process. This posterior distribution will be a better candidate when used as a model parameter distribution for the patient-specific G\&R simulation.

The Bayesian approach, however, is less helpful when there is no consensus about what the prior distribution should be. We know that prior information is a key part of the Bayesian inference and represents information about the uncertain parameters that are combined with the probability distribution of new data to yield the posterior distribution, which in turn is used for future inferences and the related decisions. In general, the key issues in setting up a prior distribution are: (1) information used to construct the prior distribution, and (2) properties of the resulting posterior distribution (conjugate family). Here, we treated the nonmeasurable (estimated) model parameters in this statistical model as random variables. Since we know the age and gender of the patients to whom the biaxial test data is related, we can update our prior knowledge using the conditional joint distribution of parameters. So, if we want to achieve the results from Bayesian calibration, the preliminary step should be achieving our main goal of constructing the prior distribution of the parameters. An overview of our approach in building the prior distribution is illustrated in Fig. 3.

2.4 Correlation Study and Joint Distribution. Recently, more papers have reported the potential relation between the mechanical behavior of aortic tissues with subject-specific information such as age, health conditions, genetics, and diet [47]. Although associated with other individual characteristics (e.g., cigarette smoking, having vascular diseases, genetics) $[48,49]$, gender and age are considered to be the basic but most relevant available variables for arterial mechanics. Therefore, using the experimental data, we study the correlation between the estimated parameters with age and gender. The framework can be, however, general enough so that the other variables can be implicated in specific applications. The population Pearson correlation study is widely used in statistics to find the correlation (linear dependency) between two sets of data. We assume that the data sets are correlated if the correlation coefficient $|\rho|>0.3$ and the $P$-value $<0.1$. For the calculation of $\rho$ and $P$-value, R software is used.

We assume that the best-fitted values of parameters are the replications of random variables with an unknown distribution. Our goal is to find the best distribution that can fit to the estimated parameters. Considering the correlation of each parameter with age and gender, we divide our 11 parameters into two groups: Group-I with parameters that show correlations with age or gender and Group-II with parameters having no correlation with age and gender.

For Group-I, a regression between parameter values and age is used. For simplicity of regression, and by investigating the estimated parameters we choose two terms age and 1/age. The regression model used for the parameter $X_{I}$ from Group-I is given by

$$
X_{I}=\alpha+\beta_{1} \cdot \text { age }+\frac{\beta_{2}}{\text { age }}+\varepsilon
$$

where $\varepsilon$ is the standard normal distribution, $\varepsilon \sim N\left(0, \sigma^{2}\right)$. For regression of each parameter, we observe the $R^{2}$ value, the $P$ value of the regression, and the $P$-value of each coefficient $\left(\alpha, \beta_{1}, \beta_{2}\right)$. Although both terms (age, $1 /$ age) in the regression model could be used in which $R^{2}$ became maximum, we found that for most cases (based on our observations) using just one term sufficiently described the relation of the parameter with age, and we do not need to have all terms in our regression equation. Based on the regression model introduced in Eq. (20), parameter $X_{I}$ has the conditional normal distribution as

$$
X_{I} \sim N\left(\alpha+\beta_{1} \cdot \text { age }+\frac{\beta_{2}}{\text { age }} \mid \text { age, } \sigma^{2}\right)
$$

For Group-II, we investigated distributions via transformations in order to find the best distribution for each parameter. In order to find which original distribution or transformed distribution provides a better representation of a specific parameter, we use the Anderson-Darling statistic (AD) and $P$-values. Between the two distributions, the one with smaller $\mathrm{AD}$ and higher $P$-value is a better candidate. Using MINITAB (Minitab Inc., State College, PA), we compare the $\mathrm{AD}$ and $P$-value of the original and transformed distribution for each parameter in Group-II: Box-Cox and Johnson transformations, normal, lognormal, three-parameter lognormal, exponential, two-parameter exponential, Weibull, three-parameter Weibull, gamma, three-parameter gamma, logistic, loglogistic, and three-parameter loglogistic distributions. 
Using the Box-Cox transformation, we transform each of the parameters in Group-II $\left(X_{I I}\right)$ to new parameters $(Y)$. The Box-Cox transformation is one of the useful transformations that tends to make data more normally distributed. However, this is not guaranteed [50] and the results of the transformation should be evaluated on whether they have normal distributions or not. The Box-Cox transformation function is given by

$$
Y=\left\{\begin{array}{cc}
\frac{X_{I I}^{\gamma}-1}{\gamma} & \text { for } \gamma \neq 0 \\
\log X_{I I} & \text { for } \gamma=0
\end{array}\right.
$$

where $\gamma$ is the transformation parameter. Based on the value for $\gamma$, the distribution for $Y$ would be different. Using the loglikelihood function, we find the optimum value for $\gamma$ so that $Y$ has the smallest standard deviation. However, this does not guarantee normality and we must check whether the normal distribution assumption for each parameter $(Y)$ is acceptable. A hypothesis test with the null hypothesis that the transformed parameter $(Y)$ has a normal distribution can determine whether our assumption for normality is valid. The hypothesis is tested for

$$
\begin{array}{ll}
H_{0}: & H_{1}: \\
Y \text { has normal distribution } & Y \text { does not have normal distribution }
\end{array}
$$

If the $P$-value is large enough $(P$-value $>0.05)$, we fail to reject the null hypothesis and assumption of normality is acceptable (but not proved). We fit a normal distribution to the transformed parameter $Y$. So

$$
Y \sim N\left(\mu, \tau^{2}\right)
$$

where $\mu$ and $\tau$ are the mean and standard deviation of the fitted normal distribution. Using the fitted normal distribution combined with the Box-Cox transformation function, the distribution of the original parameter $X_{I I}$ would be

$$
f_{X_{I I}}\left(x_{I I}\right)=\frac{1}{\tau \sqrt{2 \pi}} \exp \left\{-\frac{\left[y\left(x_{I I}\right)-\mu\right]^{2}}{2 \tau^{2}}\right\}
$$

where $y\left(x_{I I}\right)$ is either equal to $\left(x_{I I}^{\gamma-1} / \gamma\right)$ or to $\log x_{I I}$, depending on the result from our analysis using Eq. (22).

The final step is to construct the joint distribution of the transformed parameters $Y$ of Group-II and the parameters of Group-I, $X_{I}$. We combine these two groups and rename these new parameters as $Z$ (just for representing purposes).

Now considering that all the parameters $\left(Z_{1}, Z_{2}, \ldots, Z_{11}\right)$ have normal distributions $\left(X_{I}\right)$ or conditional normal distributions $(Y)$, we build the multivariate normal distributions by calculating the sample covariance matrix as

$$
\bar{\Sigma}_{i j}=\nu_{i j} \sigma_{i} \sigma_{j}
$$

where $\Sigma_{i j}$ is the element in the $i$ th row and $j$ th column of the sample covariance matrix $\bar{\Sigma}$. $\nu_{i j}$ is the correlation coefficient between $Z_{i}$ and $Z_{j}$ and $\sigma_{i}$ is the standard deviation of $Z_{i}$. Finally, we construct the multivariate normal distribution as [51]

$$
P_{\boldsymbol{Z}}\left(z_{1}, z_{2}, \ldots, z_{11}\right)=\frac{1}{\sqrt{(2 \pi)^{11}|\overline{\boldsymbol{\Sigma}}|}} \exp \left(-\frac{1}{2}(\boldsymbol{Z}-\overline{\boldsymbol{\mu}})^{\mathrm{T}} \overline{\boldsymbol{\Sigma}}^{-1}(\boldsymbol{Z}-\overline{\boldsymbol{\mu}})\right)
$$

where $\overline{\boldsymbol{\mu}}$ is the mean vector $\overline{\boldsymbol{\mu}}=\left[\mu_{1}, \mu_{2} \ldots, \mu_{11}\right], \mu_{i}$ is the mean of $Z_{i}$ for which the correlated parameters with age is a function of age, Eq. (20), and for which the rest of the parameters is a constant value, Eq. (24). The $(1-\omega) 100 \%$ confidence interval for the mean vector $\overline{\boldsymbol{\mu}}$ is given by [51]

$$
k(\overline{\boldsymbol{\mu}}-\boldsymbol{Z})^{\mathrm{T}} \overline{\boldsymbol{\Sigma}}^{-1}(\overline{\boldsymbol{\mu}}-\boldsymbol{Z}) \leq \frac{(k-1) l}{k-l} F_{l, k-l}(\omega)
$$

where $k$ is the sample size and $l$ is the dimension of the constructed multivariate normal distributions. In our problem $k=17$ and $l=11 . F_{l, k-l}(\omega)$ is the $\omega$ critical value of the $F$-distribution, $l$ is the degree-of-freedom in the numerator, and $k$ is the degree-offreedom in the denominator. Any vector of $\boldsymbol{Z}$ that satisfies the above inequality would be in the $(1-\omega) 100 \%$ confidence interval of the mean vector $\bar{\mu}$.

\section{Results}

3.1 Best-Fit Values. Using nonlinear regression, we estimated the parameters for 17 specimens. The $R^{2}$ values revealed a good fit between the theoretical values and estimated values from the experimental measurement for the Cauchy stress $\left(R_{\mathrm{avg}}^{2}=0.954\right)$. Estimated values for parameters are provided in Table 2 .

In order to see the variation with respect to age in the mechanical behavior of the aorta, two samples of fitting plots for a 19-year-old male and a 78-year-old female for both circumferential and axial data are shown in Fig. 4. The dotted lines show the experimentally measured data and the solid lines show the theoretically calculated values using the best-fit parameters from Table 2. Age dependency of the aorta material behavior is well illustrated in Fig. 4. Figures 4(a) and 4(b), which represent the 19 -year-old male's data show more extensible and linear material behavior in comparison to Figs. $4(c)$ and $4(d)$, which illustrate stiffer and more exponential shape behavior.

3.2 Correlation. Table 3 shows the correlation coefficients $\rho$ and $P$-values for the correlation study between parameters with age and gender. Results show that parameters do not have any correlation with the gender of patients. Considering this, we can combine information of both genders into one category to construct the joint conditional distribution of all parameters. We found moderate to strong correlations of parameters with age. The elastin mass fraction, $\phi^{e}$, shows a very strong negative correlation with age $(\rho=-0.760, P=0.0000<0.1)$.

3.3 Fitting of Parameter Distributions. Based on the results from Table $3, c_{m_{1}}, G_{h}^{m}, G_{1}^{e}, G_{1}^{e}$, and $\alpha$ which did not show any correlation are categorized as Group-II, and $c_{e}, c_{k_{1}}, c_{k_{2}}, c_{m_{2}}$, $G_{h}^{c}$, and $\phi^{e}$ are categorized as Group-I.

Table 4 provides the results of the regression for the parameters in Group-I. In the regression analysis, the regression $P$-value is based on the null hypothesis that the residuals do not follow normal distribution (where the residuals are the differences between the real values for the parameter at given ages of specimens subtracted by the predicted values using the regression relation). Hence, smaller regression $P$-values indicate the reliability of the regressions, and as it has been listed in Table 4, all the regression $P$-values are smaller than 0.1 . All coefficient $P$-values listed in Table 4 are smaller than 0.1 except in two cases where they are 0.308 and 0.274 for $c_{m_{2}}$ and $c_{k_{2}}$, respectively. Although high coefficient $P$-values will generally result in higher regression $P$-values, as illustrated in Table 4 , regression $P$-values of $c_{k_{2}}$ and $c_{m_{2}}$ are still small enough to indicate a reliable regression. $R^{2}$ of the regressions are also listed in Table 4. Looking over $R^{2}$ in the table, we found that they were not very close to 1 , but this did not indicate that regression results were not valuable or trustworthy. In this regression study, we were able to model the changes of parameters due to the changes in the age of the patient, but some other information has been missed due to our lack of knowledge about the patient's condition. In this regression study, the $R^{2}$ value indicates the portion $\left(R^{2}\right)$ of the change in the parameter value (response) with the possibility to be captured by the regression model, age (predictor), and what portion $\left(1-R^{2}\right)$ of the change is modeled by normal error $(\varepsilon)$. 
Table 2 Best-fit parameters for the constitutive mixture model

\begin{tabular}{|c|c|c|c|c|c|c|c|c|c|c|c|c|c|c|}
\hline No. & $\begin{array}{l}\text { Age } \\
(\mathrm{yr})\end{array}$ & Gender & $\begin{array}{c}c_{e} \\
\left(\mathrm{~J} \mathrm{~kg}^{-1}\right)\end{array}$ & $\begin{array}{c}c_{k_{1}} \\
\left(\mathrm{~J} \mathrm{~kg}^{-1}\right)\end{array}$ & $c_{k_{2}}$ & $\begin{array}{c}c_{m_{1}} \\
\left(\mathrm{~J} \mathrm{~kg}^{-1}\right)\end{array}$ & $c_{m_{2}}$ & $G_{1}^{e}$ & $G_{2}^{e}$ & $G_{h}^{m}$ & $G_{h}^{c}$ & $\phi^{e}$ & $\begin{array}{c}\alpha \\
(\operatorname{deg})\end{array}$ & $R^{2}$ \\
\hline 1 & 19 & M & 51.90 & 78.56 & 0.67 & 11.72 & $3.25 \times 10^{-6}$ & 1.459 & 1.370 & 1.237 & 1.351 & 0.302 & 47.0 & 0.994 \\
\hline 2 & 22 & $\mathrm{~F}$ & 56.22 & 672.18 & 2.54 & 62.96 & 2.37 & 1.431 & 1.306 & 1.062 & 1.104 & 0.497 & 43.7 & 0.994 \\
\hline 3 & 23 & $\mathrm{M}$ & 31.37 & 57.26 & 0.40 & 44.17 & 3.48 & 1.809 & 1.335 & 1.080 & 1.451 & 0.266 & 41.9 & 0.992 \\
\hline 4 & 25 & M & 60.49 & 77.81 & 0.92 & 0.31 & 3.20 & 1.368 & 1.434 & 1.392 & 1.354 & 0.304 & 40.7 & 0.989 \\
\hline 5 & 32 & M & 75.20 & 1415.60 & 5.14 & 0.28 & 10.82 & 1.273 & 1.359 & 1.256 & 1.054 & 0.214 & 47.8 & 0.983 \\
\hline 6 & 35 & $\mathrm{~F}$ & 33.76 & 558.05 & 5.95 & 197.48 & 4.16 & 1.784 & 1.016 & 1.023 & 1.107 & 0.263 & 43.4 & 0.866 \\
\hline 7 & 39 & M & 57.43 & 966.44 & 8.83 & $3.06 \times 10^{-3}$ & 33.94 & 1.391 & 1.389 & 1.223 & 1.070 & 0.078 & 46.2 & 0.927 \\
\hline 8 & 47 & $\mathrm{M}$ & 93.51 & 689.78 & 16.22 & 15.06 & $4.91 \times 10^{-9}$ & 1.198 & 1.303 & 1.200 & 1.078 & 0.083 & 45.1 & 0.941 \\
\hline 9 & 47 & $\mathrm{~F}$ & 66.757 & 527.54 & 9.8063 & 0.88 & $2.51 \times 10^{-13}$ & 1.304 & 1.510 & 1.955 & 1.096 & 0.156 & 47.1 & 0.984 \\
\hline 10 & 47 & M & 99.66 & 1762.60 & 2.15 & 1.64 & 26.90 & 1.206 & 1.204 & 1.146 & 1.048 & 0.295 & 45.9 & 0.978 \\
\hline 11 & 50 & $\mathrm{~F}$ & 157.75 & 3292.20 & $7.48 \times 10^{-7}$ & 20.06 & $1.47 \times 10^{-13}$ & 1.102 & 1.169 & 1.163 & 1.028 & 0.214 & 45.9 & 0.961 \\
\hline 12 & 61 & $\mathrm{M}$ & 75.99 & 1484.27 & 15.61 & $2.19 \times 10^{-6}$ & 2.64 & 1.274 & 1.301 & 1.801 & 1.052 & 0.076 & 43.0 & 0.966 \\
\hline 13 & 66 & M & 73.07 & 1425.11 & 7.45 & 54.50 & $3.73 \times 10^{-13}$ & 1.316 & 1.186 & 1.073 & 1.056 & 0.203 & 44.5 & 0.948 \\
\hline 14 & 69 & M & 253.2 & 1958.9 & 99.062 & 3.14 & $2.85 \times 10^{-13}$ & 1.054 & 1.109 & 1.521 & 1.033 & 0.101 & 41.6 & 0.966 \\
\hline 15 & 71 & M & 31.17 & 1927.70 & 6.77 & 0.32 & 121.41 & 1.790 & 1.489 & 1.095 & 1.039 & 0.036 & 50.7 & 0.871 \\
\hline 16 & 75 & $\mathrm{~F}$ & 63.73 & 2529.92 & $2.03 \times 10^{-20}$ & 0.47 & 173.92 & 1.365 & 1.214 & 1.079 & 1.031 & 0.063 & 51.7 & 0.874 \\
\hline 17 & 78 & $\mathrm{~F}$ & 113.94 & 887.82 & 60.41 & $3.86 \times 10^{-3}$ & 2.93 & 1.189 & 1.111 & 1.578 & 1.052 & 0.069 & 44.0 & 0.985 \\
\hline \multirow{2}{*}{\multicolumn{3}{|c|}{$\begin{array}{l}\text { Median } \\
\text { Mean }\end{array}$}} & 66.8 & 966.4 & 6.8 & 7.4 & 4.2 & 1.316 & 1.303 & 1.200 & 1.056 & 0.203 & 45.1 & - \\
\hline & & & 82.1 & 1194.8 & 16.1 & 29.5 & 35.1 & 1.371 & 1.283 & 1.117 & 1.287 & 0.189 & 45.3 & - \\
\hline
\end{tabular}

(a)

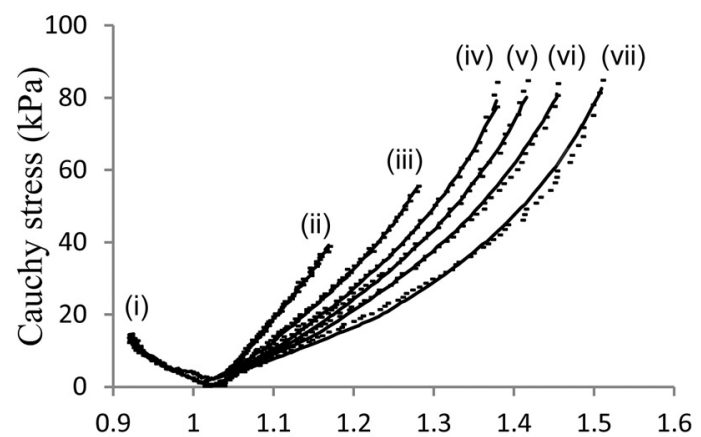

(c)

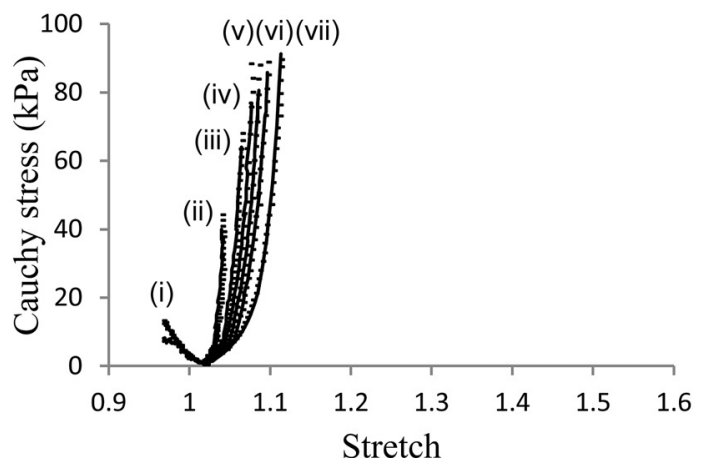

(b)

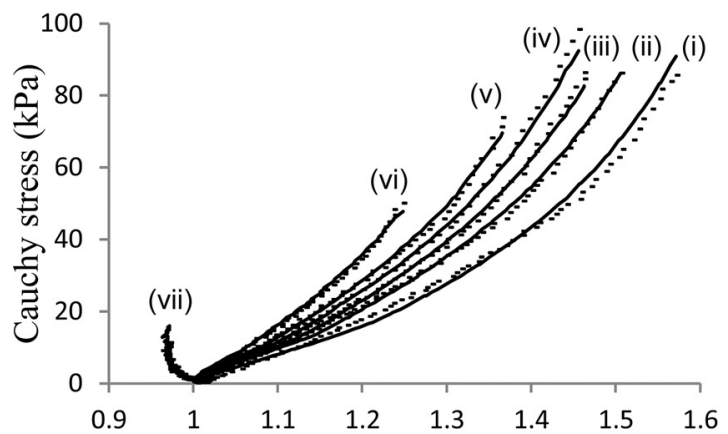

(d)

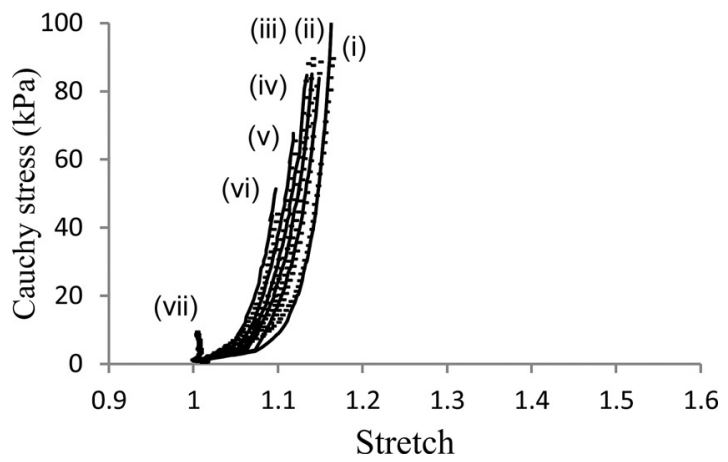

Fig. 4 Stress-stretch curves for experimental results (dots) from Vande Geest et al. [40,46], and best-fit constitutive model (lines) for seven biaxial tension controlled protocols with $T_{z z}: T_{\theta \theta}$ equal to (i) $0.1: 1$, (ii) $0.5: 1$, (iii) $0.75: 1$, (iv) $1: 1$, (v) $1: 0.75$, (vi) 1:0.5, and (vii) 1:0.1. (a) and $(b)$ Nonaneurysmal AA of a 19-year-old male axial and circumferential, respectively. (c) and (d) Nonaneurysmal AA of a 78-year-old female axial and circumferential, respectively.

Table 3 Correlation coefficients and $P$-values of the parameters' correlation with age and gender. When $|\rho|>0.3$ and a $P$-value $<0.1$, the parameters are correlated with age and gender (indicated in bold face).

\begin{tabular}{|c|c|c|c|c|c|c|c|c|c|c|c|c|}
\hline & & $c_{e}$ & $c_{k_{1}}$ & $c_{k_{2}}$ & $c_{m_{1}}$ & $c_{m_{2}}$ & $G_{h}^{c}$ & $G_{h}^{m}$ & $G_{1}^{e}$ & $G_{2}^{e}$ & $\phi^{e}$ & $\alpha$ \\
\hline Age & $\begin{array}{l}\rho \\
P \text {-value }\end{array}$ & $\begin{array}{l}0.409 \\
0^{2.103}\end{array}$ & $\begin{array}{l}0.526 \\
0.007\end{array}$ & $\begin{array}{l}0.502 \\
0.040\end{array}$ & $\begin{array}{r}-0.278 \\
0.280\end{array}$ & $\begin{array}{l}0.455 \\
0.066\end{array}$ & $\begin{array}{r}-0.685 \\
0.002\end{array}$ & $\begin{array}{r}-0.234 \\
0.366\end{array}$ & $\begin{array}{r}-0.331 \\
0.194\end{array}$ & $\begin{array}{r}-0.326 \\
0.201\end{array}$ & $\begin{array}{r}-0.760 \\
0.000\end{array}$ & $\begin{array}{l}0.315 \\
0.218\end{array}$ \\
\hline Gender & $\begin{array}{l}\rho \\
P \text {-value }\end{array}$ & $\begin{array}{l}0.001 \\
0.998\end{array}$ & $\begin{array}{r}-0.182 \\
0.485\end{array}$ & $\begin{array}{l}0.033 \\
0.901\end{array}$ & $\begin{array}{r}-0.351 \\
0.167\end{array}$ & $\begin{array}{r}-0.122 \\
0.640\end{array}$ & $\begin{array}{l}0.280 \\
0.276\end{array}$ & $\begin{array}{r}-0.063 \\
0.811\end{array}$ & $\begin{array}{l}0.029 \\
0.912\end{array}$ & $\begin{array}{l}0.337 \\
0.185\end{array}$ & $\begin{array}{r}-0.128 \\
0.626\end{array}$ & $\begin{array}{r}-0.170 \\
0.513\end{array}$ \\
\hline
\end{tabular}

${ }^{\mathrm{a}} P$-value for $c_{e}$ is not smaller than 0.1 but because it is very close to 0.1 , and also considering the value for $\rho$ we accept that $c_{e}$ has correlation with age. 
Table 4 Regression results for parameters in Group-I

\begin{tabular}{lccrrr}
\hline \hline Parameter & $\alpha(P$-value $)$ & $\beta_{1}(P$-value $)$ & $\beta_{2}(P$-value $)$ & Regression $P$-value \\
\hline$c_{e}$ & $128.2(0.000)$ & 0 & $-1792(0.093)$ & 0.097 \\
$c_{k_{1}}$ & $2420.1(0.000)$ & 0 & $-47,654(0.004)$ & 0.004 & 0.17 \\
$c_{k_{2}}$ & $-17.1(0.274)$ & $0.661(0.04)$ & 0 & 0.040 & 0.066 \\
$c_{m_{2}}$ & $-30.7(0.308)$ & $1.13(0.066)$ & 0 & 0.94 \\
$G_{h}^{c}$ & $0.904(0.000)$ & $0.30(0.000)$ & 0.000 \\
$\phi^{e}$ & $4.14 \times 10^{-1}(0.000)$ & $-4.7 \times 10^{-3}(0.000)$ & 0 & 0.25 & 0.000 \\
\hline \hline
\end{tabular}

Table 5 Box-Cox transformation results and fitted normal distribution to the transformed parameters. $P$-value ${ }^{b}$ indicates that normality test has been performed on original transformation (before transformation), and $P$-value ${ }^{a}$ indicates that the normality test has been done on best fit values after transformation.

\begin{tabular}{|c|c|c|c|c|c|c|}
\hline \multirow[b]{2}{*}{ Parameter } & \multirow[b]{2}{*}{$\gamma$} & \multirow[b]{2}{*}{ Transformation function (or transformed parameter) } & \multicolumn{4}{|c|}{ Fitted normal distribution } \\
\hline & & & Mean & Standard deviation & $P$-value ${ }^{\mathrm{b}}$ & $P$-value ${ }^{\text {a }}$ \\
\hline$c_{m_{1}}$ & 0.122 & $c_{m_{1}}^{\prime}=\frac{\left(c_{m_{1}}\right)^{0.122}-1}{0.122}$ & 0.321 & 4.82 & $<0.005$ & 0.081 \\
\hline$G_{h}^{m}$ & -3 & $G_{h}^{m^{\prime}}=\frac{1-\left(G_{h}^{m}\right)^{-3}}{3}$ & 0.145 & 0.083 & $<0.005$ & 0.359 \\
\hline$G_{1}^{e}$ & -2 & $G_{1}^{e^{\prime}}=\frac{1-\left(G_{1}^{e}\right)^{-2}}{2}$ & 0.215 & 0.084 & 0.027 & 0.603 \\
\hline$G_{2}^{e}$ & - & - & 1.283 & 0.139 & 0.886 & - \\
\hline$\alpha$ & - & - & 45.31 & 3.004 & 0.718 & - \\
\hline
\end{tabular}

Table 6 Parameters' mean value and $90 \%$ confidence intervals for 3 different ages of 45 , 60 , and 75 years old. The confidence interval for each parameter is shown outlined in brackets.

\begin{tabular}{|c|c|c|c|c|c|c|c|}
\hline \multirow{2}{*}{$\begin{array}{l}\text { Source } \\
\text { Age (yr) }\end{array}$} & \multicolumn{3}{|c|}{ Mean value [confidence interval] from current study } & \multicolumn{4}{|c|}{ Wilson et al. [6] } \\
\hline & 45 & 60 & 75 & 47 & 50 & 66 & 69 \\
\hline$c_{e}$ & $88.38[66.74,110.02]$ & $98.33[76.69,119.97]$ & $104.31[82.67,125.95]$ & 74 & 87 & 72 & 101 \\
\hline$c_{k_{1}}$ & $1361.12[1064.73,1656.51]$ & $1625.87[1329.48,1922.25]$ & $1784.71[1488.33,2081.10]$ & 1747 & 2667 & 1136 & 2610 \\
\hline$c_{k_{2}}$ & $12.65[2.78,22.51]$ & $22.56[12.69,32.43]$ & $32.48[22.61,42.34]$ & 10.6 & 16.0 & 11.2 & 20.8 \\
\hline$c_{m_{1}}$ & $73.92\left[6 \times 10^{-5}, 2500\right]$ & $73.92\left[6 \times 10^{-5}, 2500\right]$ & $73.92\left[6 \times 10^{-5}, 2500\right]$ & 24.2 & 30.9 & 15.2 & 40.4 \\
\hline$c_{m_{2}}$ & $20.15[1.09,39.21]$ & $37.10[18.04,56.16]$ & $54.05[34.99,73.11]$ & 11.8 & 11.7 & 11.4 & 12.9 \\
\hline$G_{1}^{e}$ & $1.382[1.088,1.847]$ & $1.382[1.088,1.847]$ & $1.382[1.088,1.847]$ & 1.29 & 1.18 & 1.34 & 1.19 \\
\hline$G_{2}^{e}$ & $1.283[1.224,1.342]$ & $1.283[1.224,1.342]$ & $1.283[1.224,1.342]$ & 1.30 & 1.29 & 1.25 & 1.21 \\
\hline$G_{h}^{c}$ & $1.088[1.054,1.123]$ & $1.042[1.008,1.077]$ & $1.015[0.980,1.049]$ & 1.05 & 1.03 & 1.06 & 1.03 \\
\hline$G_{h}^{m}$ & $1.269[1.011,1.870]$ & $1.269[1.011,1.870]$ & $1.269[1.011,1.870]$ & 1.10 & 1.10 & 1.10 & 1.11 \\
\hline$\phi^{e}$ & $0.203[0.168,0.237]$ & $0.132[0.097,0.167]$ & $0.062[0.027,0.097]$ & 0.30 & 0.22 & 0.23 & 0.08 \\
\hline$\alpha$ & $45.31[44.04,46.58]$ & $45.31[44.04,46.58]$ & $45.31[44.04,46.58]$ & \multicolumn{4}{|c|}{ fixed at 45.00} \\
\hline
\end{tabular}

Between all the examined distributions and transformations (mentioned in Sec. 2.4), the Box-Cox transformation was the best choice based on the AD and $P$-values for all five parameters in Group-II. Table 5 illustrates the optimum values for the transformation parameter, $\gamma$, for each parameter in Group-II. Using the maximum likelihood estimator, we found $\gamma$ so that the transformed parameters had the smallest standard deviation. Information (mean and standard deviation) regarding the fitted normal distributions to the transformed parameters, $Y$, is also provided in Table 5 with corresponding $P$-values. In the table, we have listed two columns for $P$-values, for two normality tests. $P$-value ${ }^{\mathrm{b}}$ indicates that the normality test has been performed on the original best-fit values, and $P$-value ${ }^{\mathrm{a}}$ demonstrates that the normality test has been performed on the transformed values. $P$-values that are less than 0.1 lead to the rejection of the null hypothesis of the normal distribution. Information regarding the $P$-value ${ }^{\mathrm{b}}$ shows that original best-fit values (before transformation) for $c_{m_{1}}, G_{h}^{m}$, and $G_{1}^{e}$ do not follow the normal distribution. However, $P$-value ${ }^{\mathrm{b}}$ for $G_{2}^{e}$ and $\alpha$ are good enough that we can fit the normal distribution directly to the original best-fit values and no transformation is needed. $G_{2}^{e}$ and $\alpha$ follow the normal distributions as $G_{2}^{e} \sim N\left(1.283,0.139^{2}\right)$ and $\alpha \sim N\left(45.31,3.004^{2}\right)$. Results for the $P$-value ${ }^{\mathrm{a}}$ show that the normal distribution is an acceptable candidate for $G_{h}^{m}$ and $G_{1}^{e}$ after transformation. However, $c_{m_{1}}$ does not follow the normal distribution even after transformation. At this stage, we accept the normal distribution for $c_{m_{1}}$ after transformation, $c_{m_{1}}^{\prime}$, although the $P$-value $=0.081$ does not indicate a strong statement.

3.4 Mean Values and Confidence Range. Based on the results shown in Tables 4 and 5, we calculated the mean values and confidence intervals of all parameters for the given three ages of 45, 60, and 75 years old, shown in Table 6. This table contains 


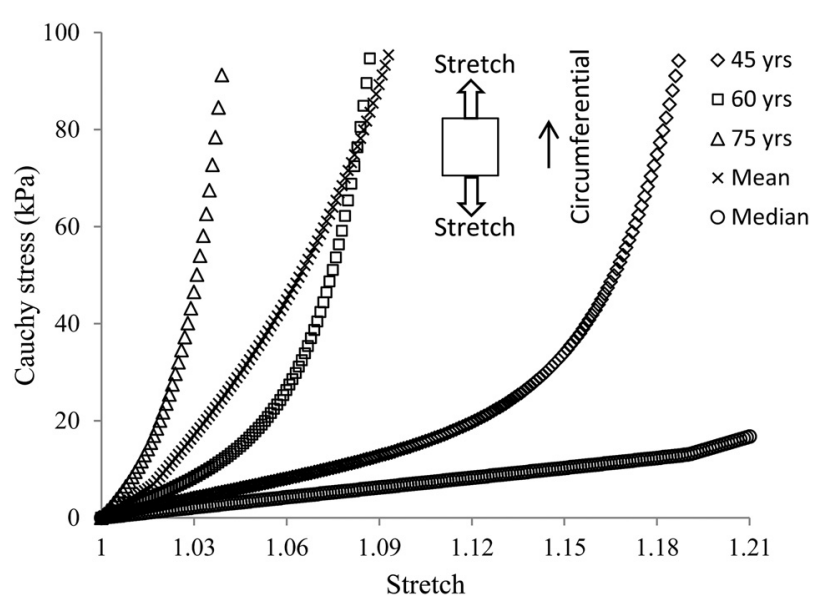

Fig. 5 Comparison between simulation results using five different parameter sets, 45,60 , and 75 years, are results of the simulation using parameters reported in Table 6. "Mean" and "Median" are the results of the simulation using the mean and median of estimations for each parameter in Table 2, respectively.

information about the effects of aging on the parameter values and the confidence intervals. Using the confidence intervals, we can compare our results with previous studies, making it easier to determine whether our findings are similar or significantly different from the literature.

As illustrated in Table 6, the mean values and the confidence intervals of $c_{e}, c_{k_{1}}, \quad c_{k_{2}}, c_{m_{2}}, G_{h}^{c}$, and $\phi^{e}$ changed for different ages due to their correlation with age. On the other hand, $c_{m_{1}}, G_{1}^{e}, G_{2}^{e}, G_{h}^{m}$, and $\alpha$ revealed no correlation with age; hence, their values remained the same for all three ages.

In the interest of comparison, we run five simulations: three simulations using values provided in Table 6 for three ages of 45, 60 , and 75 years old and two using median and mean values represented in Table 2 for model parameters. Simulation is a tension test on a square specimen under a uniaxial stretch in the circumferential direction while keeping the stretch in the axial direction constant (1.0). The stress-stretch results of those simulations have been illustrated in Fig. 5.
3.5 Multivariate Normal Distribution. In Table 6, we calculated the correlation coefficients for all parameters in $Z$ for which $c_{m_{1}}^{\prime}, G_{h}^{m \prime}$, and $G_{1}^{e \prime}$ are transformed, $G_{2}^{e}$ and $\alpha$ are the original parameters from Group-II (Table 5), and $c_{e}, c_{k_{1}}, c_{k_{2}}, c_{m_{2}}$, $G_{h}^{c}$, and $\phi^{e}$ are the original parameters from Group-I (Table 4). The correlation coefficients and $P$-values, which indicate correlation, are shown in bold face in Table 7 .

Using the correlation results between the parameters, we calculated the covariance matrix using Eq. (26). Table 7 contains information of the correlation matrix (i.e., correlation coefficients $\nu_{i j}$ ). Also, $\sigma_{i}$ and $\sigma_{j}$ are the standard deviations extracted from Tables 4 and 5. Calculated covariance matrix is illustrated in the Appendix.

The mean vector is calculated using the information of transformations and regressions from Tables 4 and 5 . In the mean vector, we used the same notation as in Table 7. In addition, we have a bar symbol used for $\bar{\mu}$ and all parameters indicating that these are sample means based on the data

$$
\overline{\boldsymbol{\mu}}=\left[\begin{array}{c}
\overline{\boldsymbol{c}}_{\boldsymbol{e}}: 128.2-\frac{1792}{\mathrm{age}} \\
\overline{\boldsymbol{c}}_{\boldsymbol{k}_{1}}: 2420.1-\frac{47,654}{\mathrm{age}} \\
\overline{\boldsymbol{c}}_{\boldsymbol{k}_{2}}:-17.1+0.661 \text { age } \\
\overline{\boldsymbol{c}}_{\boldsymbol{m}_{1}}^{\prime}: 0.321 \\
\overline{\boldsymbol{c}}_{\boldsymbol{m}_{2}}:-30.7+1.13 \text { age } \\
\overline{\boldsymbol{G}}_{1}^{\boldsymbol{e}^{\prime}}: 0.215 \\
\overline{\boldsymbol{G}}_{2}^{\boldsymbol{e}}: 1.283 \\
\overline{\boldsymbol{G}}_{\boldsymbol{h}}^{\boldsymbol{c}}: 0.904+\frac{8.3}{\mathrm{age}} \\
\overline{\boldsymbol{G}}_{\boldsymbol{h}}^{\boldsymbol{m}^{\prime}}: 0.145 \\
\bar{\phi}^{\boldsymbol{e}}: 0.414-0.0047 \mathrm{age} \\
\overline{\boldsymbol{\alpha}}: 45.31
\end{array}\right] .
$$

Using the covariance matrix, $\overline{\mathbf{\Sigma}}$, in the Appendix and the mean values vector above, the multivariate normal distribution conditioned on age was constructed using Eq. (27). Construction of this joint distribution was one of our major goals for this study. As mentioned before, this distribution was built to be utilized in the

Table 7 Correlation coefficients between all parameters $\left(Z_{i}\right)$

\begin{tabular}{|c|c|c|c|c|c|c|c|c|c|c|c|}
\hline & & $c_{k_{1}}$ & $c_{k_{2}}$ & $c_{m_{1}}^{\prime}$ & $c_{\boldsymbol{m}_{2}}$ & $G_{1}^{e \prime}$ & $G_{2}^{e}$ & $G_{h}^{c}$ & $G_{h}^{m \prime}$ & $\phi^{e}$ & $\alpha$ \\
\hline$c_{e}$ & $\underset{P \text {-value }}{\rho}$ & $\begin{array}{l}0.498 \\
0.042\end{array}$ & $\begin{array}{l}0.52 \\
0.031\end{array}$ & $\begin{array}{r}-0.13 \\
0.62\end{array}$ & $\begin{array}{c}-0.24 \\
0.346\end{array}$ & $\begin{array}{l}0.46 \\
0.062\end{array}$ & $\begin{array}{l}0.38 \\
0.123\end{array}$ & $\begin{array}{c}-0.99 \\
0\end{array}$ & $\begin{array}{c}-0.33 \\
0.195\end{array}$ & $\begin{array}{c}-0.17 \\
0.509\end{array}$ & $\begin{array}{c}-0.23 \\
0.381\end{array}$ \\
\hline$c_{k_{1}}$ & $\underset{P \text {-value }}{\rho}$ & & $\begin{array}{l}0.44 \\
0.080\end{array}$ & $\begin{array}{c}-0.21 \\
0.412\end{array}$ & $\begin{array}{l}0.43 \\
0.088\end{array}$ & $\begin{array}{l}0.97 \\
0.000\end{array}$ & $\begin{array}{c}-0.38 \\
0.131\end{array}$ & $\begin{array}{c}-0.40 \\
0.114\end{array}$ & $\begin{array}{c}-0.40 \\
0.114\end{array}$ & $\begin{array}{c}-0.58 \\
0.014\end{array}$ & $\begin{array}{l}0.46 \\
0.067\end{array}$ \\
\hline$c_{k_{2}}$ & $\underset{P \text {-value }}{\rho}$ & & & $\begin{array}{l}0.04 \\
0.882\end{array}$ & $\begin{array}{c}-0.08 \\
0.754\end{array}$ & $\begin{array}{l}0.48 \\
0.052\end{array}$ & $\begin{array}{l}0.00 \\
0.998\end{array}$ & $\begin{array}{r}-0.53 \\
0.03\end{array}$ & $\begin{array}{l}-0.39 \\
0.117\end{array}$ & $\begin{array}{c}-0.61 \\
0.009\end{array}$ & $\begin{array}{r}-0.17 \\
0.506\end{array}$ \\
\hline$c_{m_{1}}^{\prime}$ & $\underset{P \text {-value }}{\rho}$ & & & & $\begin{array}{c}-0.72 \\
0.001\end{array}$ & $\begin{array}{c}-0.29 \\
0.253\end{array}$ & $\begin{array}{c}-0.37 \\
0.142\end{array}$ & $\begin{array}{l}0.15 \\
0.556\end{array}$ & $\begin{array}{l}-0.39 \\
0.117\end{array}$ & $\begin{array}{l}0.45 \\
0.069\end{array}$ & $\begin{array}{r}-0.23 \\
0.377\end{array}$ \\
\hline$c_{m_{2}}$ & $\underset{P \text {-value }}{\rho}$ & & & & & $\begin{array}{l}0.42 \\
0.09\end{array}$ & $\begin{array}{c}-0.35 \\
0.172\end{array}$ & $\begin{array}{l}0.23 \\
0.365\end{array}$ & $\begin{array}{l}0.04 \\
0.881\end{array}$ & $\begin{array}{c}-0.51 \\
0.038\end{array}$ & $\begin{array}{l}0.40 \\
0.11\end{array}$ \\
\hline $\boldsymbol{G}_{1}^{e \prime}$ & $\underset{P \text {-value }}{\rho}$ & & & & & & $\begin{array}{c}-0.26 \\
0.319\end{array}$ & $\begin{array}{c}-0.46 \\
0.063\end{array}$ & $\begin{array}{c}-0.42 \\
0.094\end{array}$ & $\begin{array}{l}-0.67 \\
0.003\end{array}$ & $\begin{array}{l}0.44 \\
0.078\end{array}$ \\
\hline$G_{2}^{e}$ & $\underset{P \text {-value }}{\rho}$ & & & & & & & $\begin{array}{c}-0.41 \\
0.102\end{array}$ & $\begin{array}{l}0.44 \\
0.078\end{array}$ & $\begin{array}{l}0.14 \\
0.59\end{array}$ & $\begin{array}{r}-0.28 \\
0.273\end{array}$ \\
\hline$G_{h}^{c}$ & $\underset{P \text {-value }}{\rho}$ & & & & & & & & $\begin{array}{l}0.29 \\
0.254\end{array}$ & $\begin{array}{l}0.19 \\
0.459\end{array}$ & $\begin{array}{l}0.21 \\
0.424\end{array}$ \\
\hline $\begin{array}{l}G_{h}^{m \prime} \\
\phi^{e}\end{array}$ & $\begin{array}{c}\rho \\
P \text {-value } \\
\rho \\
P \text {-value }\end{array}$ & & & & & & & & & $\begin{array}{l}0.12 \\
0.644\end{array}$ & $\begin{array}{c}0.13 \\
0.631 \\
-0.34 \\
0.185\end{array}$ \\
\hline
\end{tabular}


future study which focuses on the Bayesian calibration of the G\&R model. The distribution constructed here will be used as the prior distribution.

The $90 \%$ confidence interval for all the parameters was calculated using the joint distribution. One should consider that in both the joint distribution and the confidence interval construction, we used the transformed parameters from Group-II (Table 5) and not the original parameters. The $90 \%$ confidence interval calculated as

$$
(\overline{\boldsymbol{\mu}}-\boldsymbol{Z})^{\mathrm{T}} \overline{\boldsymbol{\Sigma}}^{-1}(\overline{\boldsymbol{\mu}}-\boldsymbol{Z}) \leq 19.7
$$

where $\boldsymbol{Z}$ is the vector of parameters' values. Hence, the parameters are in the $90 \%$ confidence interval of their mean value if they satisfy the above inequality, constructed using the joint distribution. Both mean values and confidence intervals are specific to the patient through the age information.

\section{Discussion}

Ex vivo biaxial tests of human data have been of significance in accurately estimating the mechanical behavior of the AA. Stiffening of the aorta with aging was reported in multiple studies [39], emphasizing the importance of age in patient specific modeling. This study is to develop a systematic method to first estimate model parameters linked with the most important stress bearing constituents which are collagen, elastin, and smooth muscle cells. Secondarily, estimated values combined with subject-specific data, age, were used to construct conditional model parameter distributions, referred to as prior distributions. In the current study, the constructed prior distributions captured the mechanical behavior of the aorta with respect to the age of the patients. The constructed joint distribution of parameters can be directly utilized for patient-specific G\&R simulations. In previous approaches (e.g., Ferruzzi et al. [33]), they have suggested that the median of best fit values (e.g., median in Table 2) can represent the average mechanical behavior. In addition, they have reported the mean values as a representative for parameters of each age group. However, we can clearly see in Fig. 5 that the mean and median values of parameters are not able to completely capture the average mechanical behavior. The effects of age on the stress-stretch curves have been illustrated well by the current approach, which is consistent with the stiffening aorta with aging [39].

Since the model is nonlinear and the number of unknown parameters is relatively high, in order to avoid overestimation and to also enhance the computational efficiency, we applied our parameter estimation method in two steps. In the first step, we used our previous knowledge of prestretches and angle between fibers $[6,33,36,40,44]$ and fixed the values for five parameters. At the same time, we have added penalty terms to the goal function to narrow down the search area by providing a reasonable wall thickness and homeostatic stress. The second-step parameter estimation utilized the first step information while it minimized the constraints (e.g., penalty terms) to reduce the bias for ensuring local minima. The results of the final parameter estimation revealed a good fitness with the experimental stress-stretch data with an average $R^{2}$ of 0.954 while well satisfying the homeostatic conditions. Using this two-step estimation, we save a relatively good amount of time while at the same time we can decrease the uncertainty in our parameter estimation. Similar to Roccabianca et al. [40] and Ferruzzi et al. [33], the aortic wall was assumed to consist of an elastin dominated amorphous matrix and four collagen fibers with the addition of smooth muscle cells. In terms of the number of unknown parameters that were among them: ours (11), Ferruzzi et al. [33] (8), and Roccabianca et al. [40] (8). One important difference between our study and their studies is that Ferruzzi et al. $[33,34]$ did not consider prestretch for constituents, while Roccabianca et al. [40] considered prestretch but with preassigned fixed values, and in our study we considered prestretch values and estimated them using the parameter estimation method.
Although adding prestretches to the parameter estimation will increase the chance of overestimation due to the increased number of parameters, by performing the two-step parameter estimation and using the previous knowledge in the literature, the potential issue was prevented.

In the current study, the mass fraction of each parameter has been defined separate from the intrinsic material parameters. In contrast, these parameters in the two studies of Refs, $[33,40]$ have been combined together wherein, for example, the elastic parameter reported as $c$ is equivalent to $c_{e} \cdot \rho_{R}$. $\phi^{e}$ in our study. Although defining the mass fractions and material parameters separately in the strain energy function increases the number of parameters, it makes the model capable of capturing the effects of age on the constituents' intrinsic properties. Moreover, both Ferruzzi et al. [33] and Roccabianca et al. [40] considered different material parameters for collagens in different directions, $c_{1}^{i}$ and $c_{2}^{i}$, where $i=1,2,3$, and 4 , represent the circumferential, axial, and two diagonal directions. In our model, however, we assumed that the intrinsic material property for all collagen fibers in different directions were the same. Nonetheless, different specified mass fractions (with respect to the estimated mass fraction of all collagen fibers, $\left.\phi^{c}\right)$ for each direction (circumferential, axial, two diagonals) can lead to different collagen material properties in different directions of the artery.

Despite the differences between models in different studies, some comparisons can be performed. Roccabianca et al. [40] used pre-assigned values of $G_{1}^{e}=G_{2}^{e}=1.2$ and $G_{h}^{c}=1.08$. In our study, those prestretches were obtained from parameter estimation. Reported prestretches for elastin are, however, close to the mean values, $\bar{G}_{1}^{e}=1.382$ and $\bar{G}_{2}^{e}=1.283$, as well as inside and close to the constructed $90 \%$ confidence intervals, $G_{1}^{e}:[1.088,1.847], G_{2}^{e}:[1.224,1.342]$, respectively. Furthermore, for the collagen prestretch, we found a correlation with age, resulting in a nonfixed value for $G_{h}^{c}$. Based on the results of the current study, $G_{h}^{c}$ changes from 1.088 for a 45 -year-old subject to 1.015 for a 75 -year-old subject. The parameter value is, hence, close for the ages near 45 years old to that of Roccabianca et al. [40] but the value becomes smaller as the subject gets older. The angle of diagonal collagen fiber family affects the degree of anisotropy for the mechanical behavior of the artery. Ferruzzi et al. [33], Roccabianca et al. [40], and the current study estimated the mean values of $45.82 \mathrm{deg}, 45.36 \mathrm{deg}$, and $45.31 \mathrm{deg}$, respectively. Wilson et al. [6] utilized a very similar model to this study and estimated model parameters for four patients. Although they fixed the diagonal fiber's angle ( $45 \mathrm{deg}$ ), we found a consistency between our estimations and theirs. We can see that almost all of their estimated values lay in the $90 \%$ confidence intervals reported in Table 6.

Based on the correlation study, we found that $c_{e}$ and $\phi^{e}$, which are the elastin's intrinsic material property and mass fraction, have a weak positive and a strong negative correlation with age, respectively. The decrease of elastin content with aging (negative correlation of $\phi^{e}$ with aging) in the aortic wall has been reported previously in Refs. [52-54]. In addition to a reported decrease of elastin with aging, to some extent, there are considerable variations in elastin's behavior when the healthy aorta is transforming into an aneurysm. Although we have not investigated various pathological conditions in the current study, we found it interesting to mention them due to their similarity with age related changes in elastin. Rizzo et al. [55] illustrated a $92 \%$ decrease in the elastin content and a $54 \%$ increase in the collagen fiber content in the AAA wall in comparison with the healthy aorta. He et al. [56] reported histological findings of a decreased elastin content from $\phi^{e}=0.227$ in a healthy aorta to $\phi^{e}=0.024$ in an AAA. As it has been mentioned, the decrease of elastin content in the aortic wall is one of the initiators of AAA, which happens in older populations.

In addition to the decrease in mass, a reduction in crosslinks of elastin has been reported by a decrease in desmosine and isodesmosin with aging [52], which results in fragmentation of elastin. 
Fragmented elastin represents a stiffer and less extensible behavior. This behavior can be captured by the increase of $c_{e}$ in the model. We found a weak positive correlation for $c_{e}$ with aging which may correspond to the fragmentation due to aging. However, none of the two mentioned studies [33,40] have reported statistical analyses indicating whether there is any correlation between $c$ and age. Ferruzzi et al. [33] reported mean values of $c$ for three age intervals of below 30,30-60, and above 60 years old as $c=19.34,9.68$, and $3.22 \mathrm{kPa}$, respectively. Using our results, we calculated $c$ for those three intervals and the results are $c=17.58,15.82$, and $9.85 \mathrm{kPa}$. The results are comparable with the first group (less than 30 years old group), but the values are higher for the older patients. Our findings suggest that this decrease is related to the decrease of elastin's mass fraction with respect to aging (Table 2), rather than the intrinsic elastin's material parameter, $c_{e}$. As illustrated in Table $3, G_{1}^{e}$ and $G_{2}^{e}$ did not show any correlation with age. It appears that large amounts of elastin are produced during early age (neonatal period) [54], and during that period a person's aorta is growing with increased prestretches of elastin. Our finding is, however, that elastin's prestretches are not correlated with age in the range of 19-78 years old. We should mention that this is not a contradiction because the age range of our study (19-78 years) is far from the neonatal period in which most of the aorta growth happens. Therefore, our finding suggests that the properties of elastin may not be significantly changed due to age while its content (mass fraction) does.

As we report in Table 3, we found that both collagen intrinsic material parameters, $c_{k_{1}}, c_{k_{2}}$ and mass fractions $\phi^{c}$, increased with aging. This is also consistent with Ferruzzi et al. [33], reported the increase of $c_{1}^{i}$ and $c_{2}^{i}$ with aging, although there were some differences between the two models. There are less collagen fibers in the aorta during infancy and childhood, however during a process known as fibrosis these fibers start to accumulate with age. Increases of collagen fibers and its crosslink products with age have also been reported in Ref. [52].

One of the difficulties with the parameter estimations of the nonlinear model is determining whether the best-fit values represent the local or overall optimum values. This task, however, becomes more difficult when the number of unknown parameters increases. Hence, we improved our estimation by adding three penalty terms to the goal function. Although we were able to fit the simulation to the experimental results $\left(\overline{R^{2}}=0.954\right)$, we believe that more investigations are needed both experimentally and numerically to find more realistic bounds for each parameter. Even with the information that we have about each parameter's expected value, which will result in a more trustable estimation, we still need enough experimental data (experimental tests on AAAs, [57]) to better construct the distribution of each parameter.

The direction of the current research on G\&R simulation is toward enhancing the precision of estimation and the prediction capability of the mechanical behavior of the arterial wall, exploiting advanced computational models and statistical methods. A G\&R model of the AAAs, for instance, will use the conditional prior distribution combined with patient-specific data to estimate the AAA expansion rate and rupture risk. This model can be ultimately utilized by physicians in decision making for patients. Finally, we have constructed the prior distribution necessary for the future study which is the Bayesian calibration of G\&R model.

\section{Conclusion}

Toward the goal of patient-specific modeling, one critical step is to have the patient-specific model parameters. The significance of the AAA model parameters on the G\&R simulation results has been emphasized by Wilson et al. [6]. Considering the strong effect of model parameters on the G\&R simulation results, population averaged model parameters may not be the best option for patient specific modeling. In the current study, a new methodology is introduced to consider available subjects' information, age and gender, in interpreting parameter estimation results. In the fitted distributions, the effect of known factors (here age and gender) is modeled through regression and the effect of unknown or unavailable factors is modeled by the inherent randomness characteristic of the distribution. For the application of patient specific simulation, the age of the subject will be inserted in the constructed parameter model and the best estimated values with confidence intervals can be calculated. These values will be used for the patient's specific simulation.

In the current study, we considered age and gender in our correlation study but a future work can include more subject-specific information, mentioning that such information was not available to the authors of the current study. This information can be categorized in multiple groups as patient-related information like vascular diseases and genetics and specimen-related information like the location of the specimen in the aorta, thickness of the specimen, and constituent ratios. If the information is available, then it can be easily included in the distribution construction approach and build a more reliable distribution that can model parameters that are more specific to the patient.

\section{Acknowledgment}

The authors thank Drs. Jay D. Humphrey and David A. Vorp for providing the biaxial-stretching data and John S. Wilson for aiding in the modification of MATLAB codes for the parameter estimation. Authors gratefully acknowledge the support, in part, by the National Heart, Lung, and Blood Institute of the National Institutes of Health (R01HL115185 and R21HL113857) and the National Science Foundation CAREER award (CMMI-1150376). The contents are solely the responsibility of the authors and do not necessarily represent the official views of the NIH and NSF.

\section{Appendix}

The covariance matrix $(\bar{\Sigma})$ was constructed using Eq. (26)

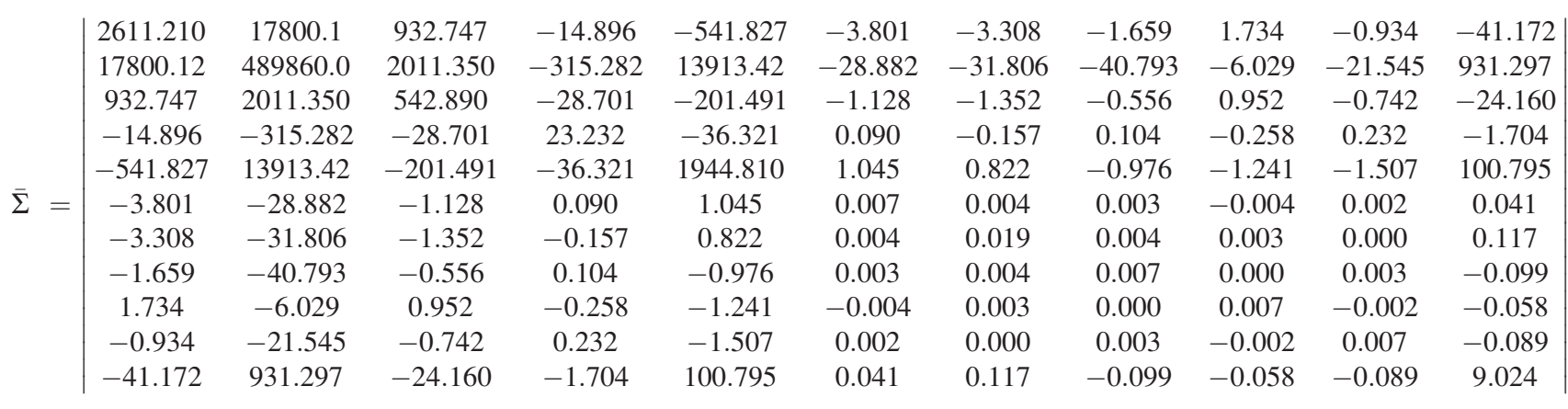




\section{References}

[1] Chien, S., Li, S., and Shyy, Y. J., 1998, "Effects of Mechanical Forces on Signal Transduction and Gene Expression in Endothelial Cells," Hypertension, 31(1 Pt 2), pp. 162-169.

[2] Guo, D.-C., Papke, C. L., He, R., and Milewicz, D. M., 2006, "Pathogenesis of Thoracic and Abdominal Aortic Aneurysms," Ann. N.Y. Acad. Sci., 1085 pp. 339-352.

[3] Humphrey, J. D., and Holzapfel, G. A., 2012, "Mechanics, Mechanobiology, and Modeling of Human Abdominal Aorta and Aneurysms," J. Biomech., 45(5), pp. 805-814.

[4] Thorne, B. C., Hayenga, H. N., Humphrey, J. D., and Peirce, S. M., 2011 , "Toward a Multi-Scale Computational Model of Arterial Adaptation in Hypertension: Verification of a Multi-Cell Agent Based Model," Front. Physiol., 2, p. 20 .

[5] Murtada, S.-I, Kroon, M., and Holzapfel, G. A., 2010, "A Calcium-Driven Mechanochemical Model for Prediction of Force Generation in Smooth Muscle," Biomech. Modell. Mechanobiol., 9(6), pp. 749-762.

[6] Wilson, J. S., Baek, S., and Humphrey, J. D., 2012, "Importance of Initial Aortic Properties on the Evolving Regional Anisotropy, Stiffness and Wall Thickness of Human Abdominal Aortic Aneurysms," J. R. Soc. Interface R. Soc., 9(74), pp. 2047-2058

[7] Upchurch, G. R., and Schaub, T. A., 2006, "Abdominal Aortic Aneurysm," Am. Fam. Physician, 73(7), pp. 1198-1204.

[8] Wilmink, T. B., Quick, C. R., Hubbard, C. S., and Day, N. E., 1999, "The Influence of Screening on the Incidence of Ruptured Abdominal Aortic Aneurysms," J. Vasc. Surg., 30(2), pp. 203-208.

[9] Assar, A. N., and Zarins, C. K., 2009, "Ruptured Abdominal Aortic Aneurysm: A Surgical Emergency With Many Clinical Presentations," Postgrad. Med. J., 85(1003), pp. 268-273.

[10] Pearce, W. H., Zarins, C. K., and Bacharach, J. M., 2008, "Atherosclerotic Peripheral Vascular Disease Symposium II Controversies in Abdominal Aortic Aneurysm Repair," Circulation, 118(25), pp. 2860-2863.

[11] Zeinali-Davarani, S., Raguin, L. G., Vorp, D. A., and Baek, S., 2011, "Identification of In Vivo Material and Geometric Parameters of a Human Aorta: Toward Patient-Specific Modeling of Abdominal Aortic Aneurysm," Biomech. Modell. Mechanobiol., 10(5), pp. 689-699.

[12] Rodríguez, J. F., Ruiz, C., Doblaré, M., and Holzapfel, G. A., 2008, "Mechanical Stresses in Abdominal Aortic Aneurysms: Influence of Diameter, Asymmetry, and Material Anisotropy," ASME J. Biomech. Eng., 130(2), p. 021023.

[13] Fillinger, M. F., Marra, S. P., Raghavan, M. L., and Kennedy, F. E. 2003, "Prediction of Rupture Risk in Abdominal Aortic Aneurysm During Observation: Wall Stress Versus Diameter," J. Vasc. Surg., 37(4), pp. 724-732.

[14] Vande Geest, J. P., Di Martino, E. S., Bohra, A., Makaroun, M. S., and Vorp, D. A., 2006, "A Biomechanics-Based Rupture Potential Index for Abdominal Aortic Aneurysm Risk Assessment," Ann. N.Y. Acad. Sci., 1085(1), pp. 11-21.

[15] Fillinger, M. F., Raghavan, M. L., Marra, S. P., Cronenwett, J. L., and Kennedy, F. E., 2002, "In Vivo Analysis of Mechanical Wall Stress and Abdominal Aortic Aneurysm Rupture Risk," J. Vasc. Surg., 36(3), pp. 589-597.

[16] Dorfmann, A., Wilson, C., Edgar, E. S., and Peattie, R. A., 2009 "Evaluating Patient-Specific Abdominal Aortic Aneurysm Wall Stress Based on Flow-Induced Loading," Biomech. Modell. Mechanobiol., 9(2), pp. 127-139.

[17] Raghavan, M. L., and Vorp, D. A., 2000, “Toward a Biomechanical Tool to Evaluate Rupture Potential of Abdominal Aortic Aneurysm: Identification of a Finite Strain Constitutive Model and Evaluation of Its Applicability," J. Biomech., 33(4), pp. 475-482.

[18] Maier, A., Gee, M. W., Reeps, C., Pongratz, J., Eckstein, H.-H., and Wall, W. A., 2010, "A Comparison of Diameter, Wall Stress, and Rupture Potential Index for Abdominal Aortic Aneurysm Rupture Risk Prediction,” Ann. Biomed. Eng., 38(10), pp. 3124-3134.

[19] Baek, S., Rajagopal, K. R., and Humphrey, J. D., 2006, "A Theoretical Model of Enlarging Intracranial Fusiform Aneurysms," ASME J. Biomech. Eng., 128(1), pp. 142-149.

[20] Watton, P. N., Hill, N. A, and Heil, M., 2004 "A Mathematical Model for the Growth of the Abdominal Aortic Aneurysm," Biomech. Modell. Mechanobiol. 3(2), pp. 98-113.

[21] Watton, P. N., and Hill, N. A., 2007, "Evolving Mechanical Properties of a Model of Abdominal Aortic Aneurysm," Biomech. Modell. Mechanobiol., 8(1), pp. 25-42.

[22] Kroon, M., and Holzapfel, G. A., 2007, "A Model for Saccular Cerebral Aneurysm Growth by Collagen Fibre Remodelling," J. Theor. Biol., 247(4), pp. 775-787.

[23] Kroon, M., and Holzapfel, G. A., 2009, "A Theoretical Model for FibroblastControlled Growth of Saccular Cerebral Aneurysms," J. Theor. Biol., 257(1), pp. 73-83.

[24] Hariton, I., deBotton, G., Gasser, T. C., and Holzapfel, G. A., 2006, "StressDriven Collagen Fiber Remodeling in Arterial Walls," Biomech. Modell. Mechanobiol., 6(3), pp. 163-175.

[25] Fung, Y. C., Fronek, K., and Patitucci, P., 1979, "Pseudoelasticity of Arterie and the Choice of Its Mathematical Expression," Am. J. Physiol., 237(5), pp. H620-H631.
[26] Takamizawa, K., and Hayashi, K., 1987, "Strain Energy Density Function and Uniform Strain Hypothesis for Arterial Mechanics," J. Biomech., 20(1), pp. 7-17.

[27] Holzapfel, G. A., Gasser, T. C., and Ogden, R. W., 2000, “A New Constitutive Framework for Arterial Wall Mechanics and a Comparative Study of Material Models," J. Elast. Phys. Sci. Solids, 61(1-3), pp. 1-48.

[28] Holzapfel, G. A., Gasser, T. C., and Ogden, R. W., 2004, "Comparison of a Multi-Layer Structural Model for Arterial Walls With a Fung-Type Model, and Issues of Material Stability," ASME J. Biomech. Eng., 126(2), pp. 264-275.

[29] Hu, J.-J., Baek, S., and Humphrey, J. D., 2007, "Stress-Strain Behavior of the Passive Basilar Artery in Normotension and Hypertension," J. Biomech., 40(11), pp. 2559-2563.

[30] Wan, W., Dixon, J. B., and Gleason, R. L., Jr., 2012, "Constitutive Modeling of Mouse Carotid Arteries Using Experimentally Measured Microstructural Parameters," Biophys. J., 102(12), pp. 2916-2925.

[31] Gasser, T. C., Ogden, R. W., and Holzapfel, G. A., 2006, "Hyperelastic Modelling of Arterial Layers With Distributed Collagen Fibre Orientations," J. R. Soc. Interface, 3(6), pp. 15-35.

[32] Zeinali-Davarani, S., Choi, J., and Baek, S., 2009, "On Parameter Estimation for Biaxial Mechanical Behavior of Arteries," J. Biomech., 42(4), pp. $524-530$

[33] Ferruzzi, J., Vorp, D. A., and Humphrey, J. D., 2011, “'On Constitutive Descriptors of the Biaxial Mechanical Behaviour of Human Abdominal Aorta and Aneurysms," J. R. Soc. Interface R. Soc., 8(56), pp. 435-450.

[34] Humphrey, J. D., and Rajagopal, K. R., 2002, "A Constrained Mixture Model for Growth and Remodeling of Soft Tissues," Math. Models Methods Appl. Sci., 12(03), pp. 407-430.

[35] Valentín, A., and Holzapfel, G. A., 2012, "Constrained Mixture Models as Tools for Testing Competing Hypotheses in Arterial Biomechanics: A Brief Survey," Mech. Res. Commun., 42, pp. 126-133.

[36] Zeinali-Davarani, S., Sheidaei, A., and Baek, S., 2011, "A Finite Element Model of Stress-Mediated Vascular Adaptation: Application to Abdomina Aortic Aneurysms," Comput. Methods Biomech. Biomed. Eng., 14(9), pp. 803-817.

[37] Sheidaei, A., Hunley, S. C., Zeinali-Davarani, S., Raguin, L. G., and Baek, S., 2011, "Simulation of Abdominal Aortic Aneurysm Growth With Updating Hemodynamic Loads Using a Realistic Geometry," Med. Eng. Phys., 33(1), pp. 80-88.

[38] Martufi, G., and Gasser, T. C., 2012, "Turnover of Fibrillar Collagen in Soft Biological Tissue With Application to the Expansion of Abdominal Aortic Aneurysms," J. R. Soc. Interface R. Soc., 9(77), pp. 3366-3377.

[39] Geest, J. P. V., Sacks, M. S., and Vorp, D. A., 2005, “Age Dependency of the Biaxial Biomechanical Behavior of Human Abdominal Aorta," ASME J. Biomech. Eng., 126(6), pp. 815-822.

[40] Roccabianca, S., Figueroa, C. A., Tellides, G., and Humphrey, J. D., 2014, "Quantification of Regional Differences in Aortic Stiffness in the Aging Human,” J. Mech. Behav. Biomed. Mater., 29, pp. 618-634.

[41] Baek, S., Valentín, A., and Humphrey, J. D., 2007, "Biochemomechanics of Cerebral Vasospasm and Its Resolution: II. Constitutive Relations and Model Simulations," Ann. Biomed. Eng., 35(9), pp. 1498-1509.

[42] Burton, A. C., 1954, "Relation of Structure to Function of the Tissues of the Wall of Blood Vessels," Physiol. Rev., 34(4), pp. 619-642.

[43] Vande Geest, J. P., Sacks, M. S., and Vorp, D. A., 2006, "The Effects of Aneurysm on the Biaxial Mechanical Behavior of Human Abdominal Aorta,' J. Biomech., 39(7), pp. 1324-1334.

[44] Zeinali-Davarani, S., Raguin, L. G., and Baek, S., 2011, "An Inverse Optimization Approach Toward Testing Different Hypotheses of Vascular Homeostasis Using Image-Based Models," Int. J. Struct. Change Solids, 3(2) pp. 33-45.

[45] Kennedy, M. C., and O'Hagan, A., 2001, "Bayesian Calibration of Computer Models," J. R. Stat. Soc. Ser. B Stat. Methodol., 63(3), pp. 425-464.

[46] Osborne, C., "Statistical Calibration: A Review," Int. Stat. Rev., 59(3), pp. 309-336.

[47] Reeps, C., Maier, A., Pelisek, J., Härtl, F., Grabher-Meier, V., Wall, W. A. Essler, M., Eckstein, H.-H., and Gee, M. W., 2012, "Measuring and Modeling Patient-Specific Distributions of Material Properties in Abdominal Aortic Aneurysm Wall," Biomech. Modell. Mechanobiol., 12(4), pp. 717-733.

[48] Enevoldsen, M. S., Henneberg, K.-A., Jensen, J. A., Lönn, L., and Humphrey, J. D., 2011, "New Interpretation of Arterial Stiffening Due to Cigarette Smoking Using a Structurally Motivated Constitutive Model," J. Biomech. 44(6), pp. 1209-1211.

[49] Forsell, C., Swedenborg, J., Roy, J., and Gasser, T. C., 2013, "The Quasi-Static Failure Properties of the Abdominal Aortic Aneurysm Wall Estimated by a Mixed Experimental-Numerical Approach,” Ann. Biomed. Eng., 41(7), pp. 1554-1566.

[50] Sakia, R. M., 1992, "The Box-Cox Transformation Technique: A Review," J. R. Stat. Soc. Ser. Stat., 41(2), pp. 169-178.

[51] Johnson, R. A., and Wichern, D. W., 2007, Applied Multivariate Statistical Analysis, 6/E, Pearson Education, New York.

[52] Sawabe, M., 2010, "Vascular Aging: From Molecular Mechanism to Clinical Significance," Geriatr. Gerontol. Int., 10(Suppl 1), pp. S213-S220.

[53] Greenwald, S. E., 2007, "Ageing of the Conduit Arteries," J. Pathol., 211(2), pp. $157-172$ 
[54] Spina, M., Garbisa, S., Hinnie, J., Hunter, J. C., and Serafini-Fracassini, A., 1983, "Age-Related Changes in Composition and Mechanical Properties of the Tunica Media of the Upper Thoracic Human Aorta," Arteriosclerosis, 3(1), pp. 64-76.

[55] Rizzo, R. J., McCarthy, W. J., Dixit, S. N., Lilly, M. P., Shively, V. P., Flinn, W. R., and Yao, J. S., 1989, "Collagen Types and Matrix Protein Content in Human Abdominal Aortic Aneurysms,” J. Vasc. Surg., 10(4), pp. 365-373.
[56] He, C. M., and Roach, M. R., 1994, "The Composition and Mechanical Properties of Abdominal Aortic Aneurysms," J. Vasc. Surg., 20(1), pp. 6-13.

[57] O'Leary, S. A., Healey, D. A., Kavanagh, E. G., Walsh, M. T., McGloughlin, T. M., and Doyle, B. J., 2014, "The Biaxial Biomechanical Behavior of Abdominal Aortic Aneurysm Tissue," Ann. Biomed. Eng., 42(12), pp. 2440-2450. 